\title{
Evaluierung des Programms Forschungskompetenzen für die Wirtschaft
}

Endbericht 
Diese Studie wurde im Auftrag des Bundesministeriums für Wissenschaft, Forschung und Wirtschaft (BMWFW) durchgeführt.

VERFASSER/IN DES BERICHTS:

Eva Heckl (Projektleitung)

Laurenz Wolf

INTERNES REVIEW/BEGUTACHTUNG:

Sonja Sheikh

LAYOUT:

Susanne Fröhlich

Die vorliegende Studie wurde nach allen Maßstäben der Sorgfalt erstellt.

Die KMU Forschung Austria übernimmt jedoch keine Haftung für Schäden oder Folgeschäden, die auf diese Studie oder auf mögliche fehlerhafte Angaben zurückgehen.

Dieses Werk ist urheberrechtlich geschützt. Jede Art von Nachdruck, Vervielfältigung, Verbreitung, Wiedergabe, Übersetzung oder Einspeicherung und Verwendung in Datenverarbeitungssystemen, und sei es auch nur auszugsweise, ist nur mit ausdrücklicher Zustimmung des Auftraggebers der Studie und der KMU Forschung Austria gestattet.

Mitglied bei:

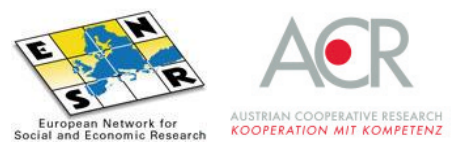




\section{Inhaltsverzeichnis}

Zusammenfassung......................................................................... 1

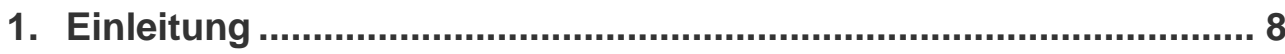

1.1. Hintergrund und Gegenstand der Evaluierung .................................. 8

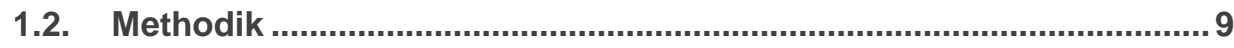

2. Das Programmkonzept......................................................................11

3. Die Programmentwicklung ................................................................16

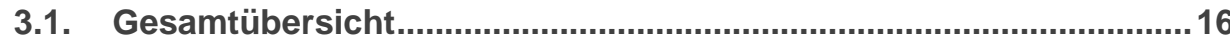

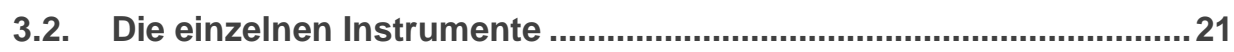

3.2.1. Qualifizierungsseminare .............................................21

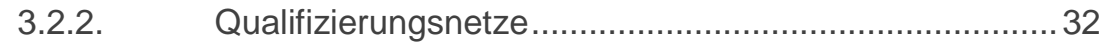

3.2.3. Innovationslehrveranstaltungen ................................ 43

4. Das Programmmanagement ............................................................53

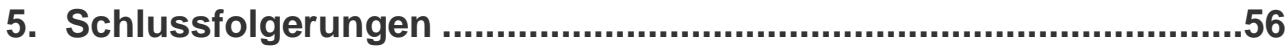




\section{Grafikverzeichnis}

Grafik 1 Logic Chart Forschungskompetenzen für die Wirtschaft .................. 12

Grafik 2 Projekt- und Teilnehmerzahlen (teilnehmende Organisationen) ........ 16

Grafik 3 Regionale Verteilung der Fördernehmer ........................................ 17

Grafik 4 Branchenverteilung der Fördernehmer, Anteil in \% ......................... 18

Grafik 5 Mehrfache Teilnahmen in Forschungskompetenzen für die

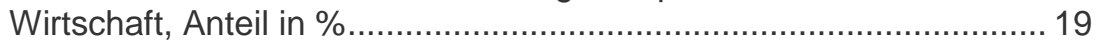

Grafik 6 Erstfördernehmer in den drei Programmlinien, Anteil in \% ............... 19

Grafik 7 Teilnahme der Unternehmen und Forschungseinrichtungen an Qualifizierungsseminaren .............................................................. 22

Grafik 8 Regionale Verteilung der Fördernehmer der Qualifizierungsseminare.

Grafik 9 TeilnehmerInnenanzahl und Frauenanteil der Qualifizierungsseminare der ersten Ausschreibung....

Grafik 10 Vorläufige Teilnehmerlnnenanzahl und Frauenanteil der Qualifizierungsseminare der zweiten Ausschreibung

Grafik 11 Teilnahme der Unternehmen und Forschungseinrichtungen an Qualifizierungsnetzen.

Grafik 12 Regionale Verteilung der Fördernehmer der Qualifizierungsnetze .... 34

Grafik 13 TeilnehmerInnenanzahl und Frauenanteil der Qualifizierungsnetze der ersten Ausschreibung....

Grafik 14 Vorläufige TeilnehmerInnenanzahl und Frauenanteil der Qualifizierungsnetze der zweiten Ausschreibung

Grafik 15 Größenklasse und Herkunft der Fördernehmer der Inno-LV ............. 44

Grafik 16 Geplante TeilnehmerInnenzahl und Frauenanteil der Innovationslehrveranstaltungen

\section{Tabellenverzeichnis}

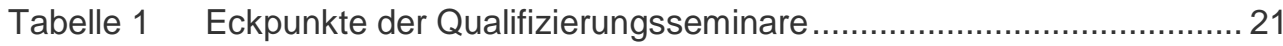

Tabelle 2 Eckpunkte der Qualifizierungsnetze ............................................... 32

Tabelle 3 Eckpunkte der Innovationslehrveranstaltungen ............................... 43 


\section{Zusammenfassung}

Der Förderschwerpunkt Forschungskompetenzen für die Wirtschaft des Bundesministeriums für Wissenschaft, Forschung und Wirtschaft (BMWFW) adressiert folgende strategischen Ziele: 1) Unternehmen im systematischen Aufbau und der Höherqualifizierung des vorhandenen Forschungs- und Innovationspersonals zu unterstützen; 2) zu einer stärkeren Verankerung unternehmensrelevanter Lehrund Forschungsschwerpunkte an österreichischen Universitäten und Fachhochschulen sowie zur Erhöhung der vielfach bemängelten sektoralen Mobilität beizutragen. Die operativen Ziele sind drei Bereichen sowie den drei Instrumenten des Förderschwerpunkts zugeordnet.

- Kompetenzaufbau: Qualifizierungsseminare als kurzfristiges, kleines Förderinstrument sollen KMU den Zugang zu FTEI-Qualifizierungsmaßnahmen erleichtern und diesen einen Überblick über für sie relevante Themenfelder geben.

- Kompetenzvertiefung: Mittelfristige, maßgeschneiderte Qualifizierungsnetze sollen zu einer Erhöhung der Innovations- und Nachfragekompetenz in zukunftsrelevanten Technologiefeldern über die Qualifizierung von MitarbeiterInnen führen.

- Kompetenzerweiterung in der angewandten Forschung: Innovationslehrveranstaltungen mit tertiärem Charakter sind längerfristige Qualifizierungsnetzwerke in neuralgischen, derzeit unterbesetzten Themenfeldern, die zur nachhaltigen Etablierung von bisher nicht adressierten, wirtschaftsnahen Themen im Qualifizierungsangebot führen sollen.

Die drei Instrumente stellen aufbauende Instrumente dar: Qualifizierungsseminare als Einsteigerformat, Qualifizierungsnetze als fortgeschrittenes Instrument und die Innovationslehrveranstaltungen mit tertiärem Charakter als Topförderung. Die Forschungsförderung erfolgt über die Qualifizierung und Weiterbildung von im Feld tätigen Personen. Damit werden Unternehmen auf Zukunftsthemen vorbereitet und ihre Fähigkeit, Innovationen aufzunehmen und umzusetzen wird verbessert. Es gibt in Österreich kein vergleichbares Programm und auch im europäischen Kontext konnte keines ähnlichen Zuschnitts im engeren Sinn gefunden werden.

\section{Programmentwicklung}

Im Rahmen des Programms, das Mitte 2011 startete, wurden bis Ende Oktober 2014553 Organisationen gefördert (448 Unternehmen und 105 Forschungseinrichtungen), wobei es eine regionale Konzentration der Fördernehmer in der Steiermark, Nieder- und Oberösterreich sowie Wien gibt. Einige der Fördernehmer haben sich an einer Programmlinie mehrfach beteiligt bzw. haben auch weitere Angebote des Förderschwerpunkts in Anspruch genommen. 
Für einen großen Teil der Fördernehmer stellte die Beteiligung an Forschungskompetenzen für die Wirtschaft die erste Unterstützung durch die FFG dar. Insgesamt fanden rd. $42 \%$ der Unternehmen und rd. $18 \%$ der Forschungseinrichtungen den Zugang zum Förderportfolio der FFG über diesen Förderschwerpunkt. Bei den Qualifizierungsseminaren waren die Hälfte der Unternehmen „FFG-Neulinge“, bei den Qualifizierungsnetzen rd. ein Drittel (36\%) und bei den Innovationslehrveranstaltungen knapp jeder Fünfte (17\%). Allen Instrumenten gelingt es, diese Einsteiger auch nachhaltig an F\&E heranzuführen, was sich an den Beteiligungen der FFG-Neulinge an weiteren durch die FFG geförderten Projekten zeigt sowie an den Folgeaktivitäten aller geförderten Unternehmen. Rd. jeder Vierte Fördernehmer nahm seit September 2013 andere FFG-Förderungen in Anspruch (v.a. im Rahmen der Basisprogramme und von Comet und Talente).

\section{Die einzelnen Instrumente}

Insgesamt wurden 38 Qualifizierungsseminare gefördert, 25 davon fielen in die erste Ausschreibung und wurden bereits abgeschlossen. In einem Qualifizierungsseminar sind durchschnittlich 5,7 Unternehmen und 2,3 Forschungseinrichtungen konzentriert. Das umfangreichste Einzelprojekt weist 22 Teilnehmer auf, demgegenüber stehen zwei Seminare, die lediglich drei Teilnehmer aufweisen. Ziel der Seminare war in den meisten Fällen die Vermittlung anwendbaren Wissens und die Kompetenzerweiterung der beteiligten Unternehmen in einem spezifischen Themenbereich. An den Seminaren selbst nahmen 374 (1. Ausschreibung) Personen teil bzw. werden laut Planung weitere 142 Personen (Stand Oktober 2014) teilnehmen (2. Ausschreibung). Etwa 14\% der Seminarteilnehmerlnnen (1. Ausschreibung) waren weiblich, in der zweiten Ausschreibung liegt der geplante Anteil derzeit bei etwa $25 \%$. Allerdings schwankt die „Frauenquote" bei den einzelnen Seminaren beträchtlich. Die Seminare beinhalteten meist einen einführenden theoretischen Teil und dann interaktive Elemente, wie z.B. Übungen am Gerät, Livepräsentationen, Werkbesichtigungen, Exkursionen, Laborübungen, etc. Die Praxisrelevanz der Seminare wurde hervorgehoben. Das Feedback der Teilnehmerlnnen, das meist nach den Schulungen eingeholt wurde (Fragebogen und/oder Feedbackgespräche), war fast durchwegs (sehr) gut.

Die Qualifizierungsseminare haben sich sowohl in ihrer Konzeption als auch in ihrer praktischen Ausgestaltung als Einsteigerformat bewährt. Auch ist der Anteil an Kleinunternehmen in diesem Format am höchsten. Die Seminare entsprechen den Bedürfnissen der Unternehmen und behandeln zukunftsrelevante Themen, da schon oft im Vorfeld Weiterbildungsbedarf bei den Unternehmen identifiziert wurde, aber auch ein mangelndes Angebot an geeigneten Qualifizierungsmaßnahmen.

Durch die Teilnahme am Qualifizierungsseminar kam es bei den Unternehmen zu einer Stärkung ihrer Innovationskompetenz, was sich eigenen Angaben zufolge in Wettbewerbsvorteilen ausdrückt. Es wurde Verständnis für Innovationsprozesse entwickelt sowie die Kommunikation mit Kundlnnen und Kooperationspartnern 
verbessert. Viele Unternehmen konnten aufbauend auf dem erworbenen Wissen und durch die Anwendung neuer Technologien ihre Produkte/Dienstleistungen verbessern bzw. ihr Produktportfolio erweitern. Auch sind weitere (geförderte) F\&E Aktivitäten initiiert worden bzw. haben die Unternehmen nun verstärkten Zugang zu wissenschaftlichen Einrichtungen. Die wissenschaftlichen Partner nützen die Erfahrungen aus den Seminaren für die Weiterentwicklung der Lehre und planen studentische Projekte in Unternehmen. Somit können durch die Qualifizierungsseminare auch Barrieren abgebaut und neue Kommunikationskanäle zwischen Wissenschaft und Wirtschaft, und insbesondere KMU, geöffnet werden.

Das Instrument der Qualifizierungsseminare ist als sehr geeignet zur Kompetenzsteigerung von KMU anzusehen. Erfolgsfaktoren sind die maßgeschneiderte Anpassung der Schulungsinhalte, der Anschluss an den aktuellsten Stand der Forschung und der Erfahrungsaustausch mit anderen TeilnehmerInnen.

Von insgesamt 21 Qualifizierungsnetzen sind 11 abgeschlossen, da diese im Rahmen der ersten Ausschreibung gefördert wurden. In den Qualifizierungsnetzwerken sind durchschnittlich 12,6 Unternehmen und 3,5 Forschungseinrichtungen zusammengefasst. Das kleinste Qualifizierungsnetzwerk weist sechs Teilnehmer auf, während das größte Netzwerk aus 6 Forschungseinrichtungen und 32 Unternehmen besteht. Die geförderten Qualifizierungsnetze verfolgten zwei wesentliche Zielbereiche. Zum einen waren dies die Steigerung der Innovationskompetenz der Unternehmen sowie der Fachkompetenz und die Verbreitung des Know-Hows (aus verwandten Branchen) durch die Vermittlung von praktisch verwertbarem Wissen bzw. methodischen Zugängen. Zum anderen wollten die Qualifizierungsnetze zu einer nachhaltigen Vernetzung zwischen Wissenschaft und Wirtschaft bzw. zwischen Unternehmen verwandter Branchen / unterschiedlicher Gewerbe zur Bearbeitung von Schnittstellenproblematiken beitragen.

Die Schulungen waren meist modular aufgebaut, wobei jedes Modul, das oft mehrere Seminare/Workshops beinhaltete, einem Thema gewidmet war. Die Seminare selbst beinhalteten meist Vorträge zur Vermittlung der Theorie, sowie Diskussionen, Gruppenarbeiten und Übungen, bei denen das Erlernte Anwendung finden konnte. Die Praxisorientierung wurde in einigen Qualifizierungsnetzen dadurch unterstützt, dass in der Ausbildung konkrete Beispiele und Projekte aus den Unternehmen bearbeitet wurden sowie das in Workshops erlangte Wissen in innerbetrieblichen Trainings umgesetzt und erprobt bzw. in einer betreuten Projektarbeit angewandt wurde. Insgesamt wurden im Zuge der ersten Ausschreibung 618 Personen ausgebildet, wovon 101 (rd. $16 \%$ ) weiblich waren. Der Anteil der Teilnehmerinnen je Projekt pendelt dabei zwischen $50 \%$ und $0 \%$ (ein Projekt). Im Rahmen der zweiten Ausschreibung wird die Qualifikation von 596 Personen bei einem etwa gleichbleibenden Frauenanteil (rd. $15 \%$ ) angestrebt. Feedback der TeilnehmerInnen wurde meist am Ende jeder Schulungseinheit oder am Ende jedes Moduls eingeholt. Dabei wurden die Kurse meist mit sehr gut und gut bewertet. 
Die Qualifizierungsnetze erweisen sich als fortgeschrittenes Instrument der Weiterbildung, das ein mittelfristiges Committment der beteiligten Partner verlangt. Unter den beteiligten Unternehmen befinden sich daher auch weniger FFG-Neulinge als bei den Qualifizierungsseminaren. Neben der Verbreiterung des Know-Hows der Projektpartner steht hier der Vernetzungsgedanke im Vordergrund. Dabei streben einige Netzwerke auch eine branchenübergreifende Zusammenarbeit an. Dies hat zur Folge, dass es zu einer größeren Heterogenität der Teilnehmerlnnen kommt. Außerdem zeigen sich Engpässe in Hinblick auf die Vereinbarkeit mit dem betrieblichen Alltag, da insbesondere kleine Unternehmen oft nur schwer ihre Fachkräfte für Weiterbildungsmaßnahmen über einen längeren Zeitraum abstellen können. So wird das Instrument von vielen Beteiligten auch als „fordernd“ bezeichnet.

Die teilnehmenden Unternehmen berichten von einer Erhöhung ihrer Innovationskompetenz, die sie teilweise durch interne Schulungen noch verstärken wollen. Dies schlägt sich u.a. in einem souveräneren Auftreten bei den Kundlnnen nieder, führt aber auch zu Motivationsschüben bei den Mitarbeiterlnnen. Auch wurden Produkte bzw. interne Unternehmensprozesse optimiert bzw. neue entwickelt. Netzwerkaktivitäten sind auch nach dem Auslaufen mancher Projekte zu beobachten, es wurden z.B. Vereine gegründet oder die Partner engagieren sich in bestehenden Netzwerken. Besonders hervorzuheben ist die nachhaltige Verankerung von Qualifizierungsangeboten (z.B. akademischer Lehrgang und Masterlehrgang, Tagesschulungen und Inhouse-Seminare), die auf Basis der Erfahrungen im Qualifizierungsnetz konzipiert wurden.

Auch die Qualifizierungsnetze genießen hohe Akzeptanz bei den Fördernehmern, was sich nicht zuletzt in der (geplanten) Teilnahme an weiteren QN-Projekten ausdrückt. Als Stärken des Instruments werden die Anpassung der Schulungsinhalte an die Bedürfnisse der Unternehmen, die Kompetenz der Vortragenden und der Vernetzungsaspekt gesehen. Auch die Möglichkeit der Diskussion mit Unternehmen anderer Branchen und die Bearbeitung von Schnittstellen sowie die Verzahnung von Wissenschaft und Praxis werden positiv hervorgehoben. Dies führt aber auch zu der Herausforderung, Unternehmen aus verschiedenen Bereichen zusammenzuführen und zu koordinieren.

Im Rahmen der bis dato einzigen Ausschreibung wurden drei Innovationslehrveranstaltungen gefördert, wobei keine zum Zeitpunkt der Evaluierung abgeschlossen ist. In den Innovationslehrveranstaltungen sind durchschnittlich 12 Unternehmen und 2,3 Forschungseinheiten zusammengefasst. Die umfangreichste Lehrveranstaltung weist 19 Beteiligungen auf, die kleinste der drei Innovationslehrveranstaltungen besteht aus 10 Teilnehmern, von denen 7 Unternehmen sind. In allen Projekten sind wissenschaftliche Partner die Konsortialführer. 
Die konkrete Ausgestaltung der Schulungen wurde zumeist im Vorfeld mit den Unternehmen diskutiert. Alle Innovationslehrveranstaltungen setzen bei den Schulungen auf eine Mischung aus Vorträgen und praktischen Übungen. Im Rahmen der drei Projekte sollen insgesamt 169 Personen qualifiziert werden, davon sind 16 (rd. $9 \%$ ) weiblich. Der Frauenanteil unter den Teilnehmerlnnen liegt in allen Projekten unter $20 \%$, die Lehrveranstaltung, die dem Bereich der IKT zuordenbar ist, wird gänzlich ohne weibliche Beteiligung durchgeführt.

Das anspruchsvollste Instrument Innovationsveranstaltungen spricht naturgemäß nur wenige Fördereinsteiger an. Die Kompetenzerweiterung erfolgt in Schlüsseltechnologien bzw. in Themenfeldern, die für die Unternehmen stark an Bedeutung gewinnen. In einem Fall steht das branchenübergreifende Systemdenken zur Bewältigung zukünftiger Herausforderungen im Vordergrund. In allen drei Fällen dürfe das jeweilige Projekt eine große Lücke im Angebot schließen, da diese Spezialthemen bzw. sehr dynamischen Bereiche nicht in Standardschulungen behandelt werden. Daher sprechen die Unternehmen auch von einem sehr attraktiven Schulungsangebot.

Die Konkretisierung der Transferprojekte, die dazu dienen sollen, das Gelernte in den Unternehmen zu implementieren, steht in den meisten Fällen noch aus ebenso wie die nachhaltige Verankerung von Schulungsangeboten. Hier gibt es erste Absichtserklärungen (z.B. Konzeption von Weiterbildungsprogrammen und Schulungen für Unternehmen, Vortrags- und Übungseinheiten können in die Lehre an der Universität übernommen werden). Eine Kompetenzerweiterung wird seitens der beteiligten Unternehmen erwartet, v.a. hinsichtlich der Entwicklung einer ganzheitlichen Sicht über Branchen hinweg. Zudem dürften die Innovationslehrveranstaltungen bestehende Kooperationen festigen.

Die bis dato durchgeführten Schulungen stoßen auf positive Resonanz der TeilnehmerInnen, die Fördernehmer begrüßen das Instrument, da hier unternehmerische Weiterbildung auf höchstem Niveau und spezialisierte Ausbildungen stattfinden können. Eine entscheidende Stärke des Instruments ist der multilaterale Know-How-Transfer (von den wissenschaftlichen Partnern zu den Unternehmen, aber auch umgekehrt sowie zwischen den Unternehmen). Damit einher geht auch die verstärkte Netzwerkbildung und informelle Zusammenarbeit.

\section{Programmmanagement}

Das Programm weicht von der Förderlogik der FFG im engeren Sinn ab, als in dessen Rahmen keine Forschungsprojekte sondern Qualifizierungsmaßnahmen gefördert werden. Es ist relativ neu und hat noch keine hohe Bekanntheit unter den potenziellen Förderwerbern. Dies hat auch Folgen in Hinblick auf die Ausschöpfung der Fördermittel, die für die Qualifizierungsseminare reservierten Budgets werden nicht verbraucht. Hier strebt das Programmmanagement eine Informationsoffensive an, um v.a. Fördereinsteiger und kleine Unternehmen besser erreichen zu können. 
Die Auswahlverfahren und Förderkriterien sind den jeweiligen Instrumenten angemessen. Hauptdiskussionspunkt in den Jurysitzungen war die Additionalitätsfrage, d.h. ob ein eingereichtes Schulungsangebot schon am Markt angeboten wird oder nicht. In diesem Bereich zeigten einige Anträge auch Schwächen. Auch die qualitative Darstellung der Qualifizierungsmaßnahmen dürfte teilweise (v.a. im Rahmen der ersten Ausschreibungen) nicht präzise erfolgt sein.

Die Juroren, Fördernehmer und Projektpartner beurteilen die Abwicklung und Betreuung durch die FFG sehr positiv. Die erfahrenen Unternehmen sprechen von üblichen Erfordernissen, die Fördereinsteiger schätzen sehr, dass die wissenschaftlichen Partner die Administration mit der FFG übernehmen können.

\section{Schlussfolgerungen}

Das Konzept des Förderschwerpunkts scheint gelungen. Die Instrumente sind für die jeweilige Zielgruppe geeignet und den jeweiligen Bedürfnissen angepasst und spiegeln den aufbauenden Charakter des Programms wider. Das Programm schließt eine Lücke im Förderportfolio der FFG, da es strukturelle Wissenstransfermaßnahmen und die Weiterbildung in Netzwerken fördert. Die einzelnen Instrumente werden von den Zielgruppen begrüßt und die bisherigen Ergebnisse lassen auf nachhaltige Wirkungen im Sinn der Programmziele schließen.

Aufgrund dieser Befunde wird eine Fortführung des Programms zum jetzigen Zeitpunkt empfohlen. Dies betrifft insbesondere die Instrumente Qualifizierungsseminare und Qualifizierungsnetze, die Innovationslehrveranstaltungen können noch nicht umfassend beurteilt werden. Erste Optimierungsvorschläge konnten wie folgt identifiziert werden:

- Teilweise sollten Zielformulierungen überdacht werden, v. a hinsichtlich der Abgrenzung von strategischen und operativen Zielen und dessen, was das Programm leisten kann. Zudem wird eine Überarbeitung der im Programmdokument aufgelisteten Indikatoren, nicht zuletzt in Hinblick auf eine weitere Evaluierung, empfohlen.

- Die Abgrenzung zwischen Qualifizierungsnetzen und Innovationslehrveranstaltungen scheint nicht ganz klar, auch aufgrund des Titels "Innovationslehrveranstaltungen“, der dem langfristigen Charakter des Instruments nicht Rechnung trägt. Hier könnte nach Alternativen gesucht werden.

- Inwieweit sich die Instrumente Qualifizierungsnetze und Innovationslehrveranstaltungen unterscheiden, steht auch mit den Wirkungen letzterer in Zusammenhang. Diese können zum gegebenen Zeitpunkt noch nicht abgeschätzt werden. Die Berechtigung dieses hoch dotierten Instruments wird davon abhängen, inwieweit es in der Folge zu einer Verankerung unternehmensrelevanter Lehr- und Forschungsschwerpunkte an Universitäten und FHs kommt, v. a. im Vergleich zu den Qualifizierungsnetzen. Auch 
den Transferprojekten wird bei der Beurteilung des Förderinstruments Bedeutung zukommen. In einer späteren Evaluierung sollten daher die Innovationslehrveranstaltungen in den Fokus gerückt werden.

- Bei den Innovationslehrveranstaltungen war den Fördernehmern nicht ganz klar, welchen Charakter Transferprojekte haben sollten. Hier sollten die Ausschreibungsunterlagen überarbeitet werden und deutlich machen, welche Art von Projekten erwartet wird.

- Bei den Qualifizierungsseminaren und auch bei den Innovationslehrveranstaltungen erwähnten einige Unternehmen, dass sie am Seminar auch ohne Förderung der Personalkosten teilgenommen hätten. In einer späteren Evaluierung sollte die Additionalitätswirkung dieser monetären Förderung systematisch im Rahmen einer Unternehmensbefragung überprüft und gegebenenfalls Anpassungen vorgenommen werden.

- Der Frauenanteil der Teilnehmerlnnen in allen drei Instrumenten spiegelt die Unterrepräsentanz von Frauen in Naturwissenschaft/Technik und Forschung im Allgemeinen und in bestimmten Branchen und Bereichen im Besonderen wider. Maßnahmen zur Steigerung der Sensibilität in Genderfragen bei den beteiligten Unternehmen könnten im Rahmen eines solchen Programms noch forciert werden.

- Die Fördermittel, die für die Qualifizierungsseminare bereit stehen, werden nicht ausgeschöpft. Da sich das Format an Fördereinsteiger richtet und auch für diese Zielgruppe sehr geeignet scheint, gilt es, zielgruppengerechte Mobilisierungsstrategien zu erarbeiten. Insbesondere Intermediäre, aber auch Interessensvertreter, können hier eine unterstützende Rolle einnehmen und zur Bekanntheit des Instruments beitragen sowie Überzeugungsarbeit in den Unternehmen leisten.

- Der Förderschwerpunkt als Ganzes ist noch nicht sehr bekannt in der Community und bei potenziellen Fördernehmern. Daher sollte insgesamt Augenmerk auf die Kommunikationsaktivitäten gesetzt werden, damit sich Forschungskompetenzen in der Wirtschaft etablieren kann. PR-Maßnahmen könnten mit den anderen Humanressourcenprogrammen der FFG koordiniert werden. Aber auch im Zusammenhang mit anderen Einsteigerformaten bzw. Formaten, die sich besonders an KMU richten, sollte das Programm in Erscheinung treten.

- Die Steigerung des Bekanntheitsgrads könnte auch durch eine bessere Vernetzung mit der Qualifizierungsszene erreicht werden. Kooperationen mit Institutionen, die die Qualifizierung von ArbeitnehmerInnen fördern (z.B. AMS), könnten weitere Kommunikationskanäle eröffnen und Synergieeffekte generieren. 


\section{Einleitung}

\subsection{Hintergrund und Gegenstand der Evaluierung}

Die gemeinsame FTI-Strategie 2020 der Bundesregierung enthält u.a. die Ziele einer nachhaltigen Reform des österreichischen Bildungssystems und der Stärkung der Innovationskraft von Unternehmen. Das erste Ziel steht im Zusammenhang mit einer Optimierung der Rahmenbedingungen für FTI sowie einer Verbesserung des Bildungs- und Innovationssystems und der Steigerung von Qualität und Quantität des Humanpotenzials für FTI in Österreich. Das zweite Ziel geht einher mit einer Intensivierung der angewandten Forschung und des Technologietransfers mit Fokus auf KMU.

Der Förderschwerpunkt Forschungskompetenzen für die Wirtschaft ${ }^{1}$ des Bundesministeriums für Wissenschaft, Forschung und Wirtschaft (BMWFW) adressiert diese Ziele und nimmt zudem die Forderung der Systemevaluierung nach einer Ausweitung des Innovationsbegriffs und der Förderung der HumanressourcenEntwicklung auf. Strategische Ziele des Programms sind vor diesem Hintergrund:

- Unternehmen im systematischen Aufbau und der Höherqualifizierung des vorhandenen Forschungs- und Innovationspersonals zu unterstützen;

- zu einer stärkeren Verankerung unternehmensrelevanter Lehr- und Forschungsschwerpunkte an österreichischen Universitäten und Fachhochschulen sowie zur Erhöhung der vielfach bemängelten sektoralen Mobilität beizutragen.

Im Rahmen des Förderschwerpunkts Forschungskompetenzen für die Wirtschaft kommen drei Instrumente zum Einsatz:

- Qualifizierungsseminare (Kompetenzaufbau)

- Qualifizierungsnetze (Kompetenzvertiefung)

- Innovationslehrveranstaltungen mit tertiärem Charakter (Kompetenzerweiterung in der angewandten Forschung)

1 Siehe: Sonderrichtlinie „Forschungskompetenzen für die Wirtschaft" des Bundesministeriums für Wirtschaft, Familie und Jugend, 27. Juni 2011 
Die Sonderrichtlinie zum Förderschwerpunkt sieht eine Evaluierung dieser neuen Instrumente vor, wobei die Module Qualifizierungsseminare und -netze aufgrund der kürzeren Laufzeit einer Schlussevaluierung ${ }^{2}$, die Innovationslehrveranstaltungen mit tertiärem Charakter einer Zwischenevaluierung unterzogen werden sollen.

Im Mittelpunkt der Evaluierung stehen das Konzept des Förderschwerpunkts, die Programmorganisation bzw. das Programmmanagement sowie die Wirkungsanalyse. Hierbei wird der Fokus auf die erste Ausschreibungsrunde gelegt. Wirkungen lassen sich teilweise aber nur abschätzen, da der Förderschwerpunkt auch auf Themen wie „Kompetenzzuwachs“ abzielt, die im Rahmen einer solchen Evaluierung nicht direkt bzw. nicht in einer so kurzen Zeitspanne (z.B. „nachhaltige Etablierung von Themen im Qualifizierungsangebot") zu erheben sind.

Der vorliegende Endbericht präsentiert die Ergebnisse der Evaluierung. Das Konzept des Förderschwerpunkts wird in Kapitel 2 analysiert, Kapitel 3 stellt die Entwicklung des Programms und der einzelnen Instrumente dar. Kapitel 4 beschäftigt sich mit dem Programmmanagement und in Kapitel 5 werden die Schlussfolgerungen präsentiert.

\subsection{Methodik}

Aufgrund der vorgegebenen sehr kurzen Laufzeit der Evaluierung wurde ein qualitativer Ansatz gewählt. Es musste auf eine quantitative Befragung aller Fördernehmer verzichtet werden. Alle Primärerhebungen im Rahmen der Evaluierung sind qualitativer Natur. Bei der Analyse der Instrumente erfolgt eine Konzentration auf die Projekte der ersten Ausschreibungen. Die angewandten Methoden werden im Folgenden kurz beschrieben:

\section{Dokumentenanalyse}

Basis der Evaluierung bildet eine ausführliche Dokumentenanalyse. Folgende Dokumente wurden analysiert:

- Das Programmdokument

- Die Ausschreibungsleitfäden

- Projektanträge (von Projekten, für die noch kein Zwischen- oder Endbericht vorliegt)

- Projektberichte (Zwischen- und/oder Endberichte)

- Protokolle der Besuche vor Ort.

Da im Rahmen der Interviews nur wenige Fördernehmer befragt werden konnten, kommt der systematischen Analyse der Projektberichte besondere Bedeutung v.a.

2 Eine Schlussevaluierung ist nur für die 1. Ausschreibung möglich, da die Projekte, die im Rahmen der 2. Ausschreibung gefördert wurden, erst am Beginn der Laufzeit stehen. 
in Hinblick auf die Ausgestaltung der Projekte, die Zusammenarbeit in den Konsortien sowie die Analyse der Wirkungen zu, um ein umfassendes Bild des Förderschwerpunkts zeigen zu können.

\section{Datenanalyse}

Zur quantitativen Erfassung des Förderschwerpunkts erfolgte eine Analyse der FFG-Monitoringdaten. Hierbei wurden alle Projekte - auch jene der zweiten Ausschreibungen - in die Analyse miteinbezogen.

\section{Interviews mit Stakeholdern}

Für die Analyse des Programmkonzepts und der Programmorganisation wurden Interviews mit Stakeholdern durchgeführt. Es erfolgten Interviews mit dem Programmeigner (BMWFW), dem Programmmanagement (FFG), der Strategieabteilung der FFG sowie mit Juroren.

\section{Interviews mit Unternehmensvertretern}

Um die Sichtweise der teilnehmenden Unternehmen genauer zu untersuchen, wurden Interviews mit leitenden Unternehmensvertretern, die in die Projekte involviert waren, durchgeführt. Es wurden 8 Interviews mit Unternehmensvertretern, die an Qualifizierungsseminaren der 1. Ausschreibung teilgenommen haben, 4 Interviews mit Unternehmensvertretern, die an Qualifizierungsnetzen der 1. Ausschreibungsrunde teilgenommen haben, und mit 7 Unternehmensvertretern, die in einer Innovationslehrveranstaltung involviert sind (mindestens 2 je Projekt), durchgeführt.

\section{Interviews mit ProjektleiterInnen}

Da für die Innovationslehrveranstaltungen noch keine Endberichte vorliegen und keines der Projekte abgeschlossen ist, wurde die Konsortialführung - in zwei Fällen im Rahmen von Besuchen vor Ort gemeinsam mit der FFG - der drei Projekte interviewt. 


\section{Das Programmkonzept}

Im Rahmen der Systemevaluierung ${ }^{3}$ wurden die Humanressourcen als Flaschenhals für die Forschung in Österreich identifiziert. Die FFG stellt immer wieder fest, dass Qualifikationen und Kompetenzen in den KMU fehlen, um an den Förderungsprogrammen teilnehmen zu können. Der Förderschwerpunkt Forschungskompetenzen für die Wirtschaft wurde daher als gezielte strukturelle Maßnahme konzipiert, um die Humanressourcen in der Wirtschaft zu stärken. Dieser Kompetenzaufbau soll in der Folge auch die Qualität der von der FFG geförderten Forschungsprojekte erhöhen.

Nachstehende Logic Chart illustriert den Förderschwerpunkt und seine Programmlogik. Die strategischen Ziele laut Programmdokument sind:

- Unternehmen im systematischen Aufbau und der Höherqualifizierung des vorhandenen Forschungs- und Innovationspersonals zu unterstützen;

- zu einer stärkeren Verankerung unternehmensrelevanter Lehr- und Forschungsschwerpunkte an österreichischen Universitäten und Fachhochschulen sowie zur Erhöhung der vielfach bemängelten sektoralen Mobilität beizutragen.

Das erste strategische Ziel entspricht der FTI-Strategie der Bundesregierung und findet sich auch in den FTI-Strategien der Bundesländer wieder. Es kann als Antwort auf die in der Systemevaluierung festgestellten Defizite verstanden werden und ist in seinem Abstraktionsgrad auf dieser Ebene optimal angesiedelt. Das strategische Ziel 2 dagegen hat einen starken operativen Charakter und kann zudem nur einem Instrument (Innovationslehrveranstaltungen) zugeordnet werden. Es ist daher als strategisches Ziel ungeeignet und sollte als operatives Ziel dem Bereich Kompetenzerweiterung bzw. den Innovationslehrveranstaltungen zugeordnet werden. Die Erhöhung der sektoralen Mobilität, d.h. der personelle Wechsel von der wissenschaftlichen in die angewandte Forschung und vice versa, kann mit dem vorliegenden Instrumentarium nicht erreicht werden und scheint auch nicht adäquat im Zusammenhang mit Qualifizierungsmaßnahmen für Unternehmen, in denen keine kooperativen Forschungsprojekte im Mittelpunkt stehen.

3 WIFO, convelop, KMU Forschung Austria, Prognos (2009): Systemevaluierung der österreichischen Forschungsförderung und -finanzierung 


\section{Grafik 1 Logic Chart Forschungskompetenzen für die Wirtschaft}
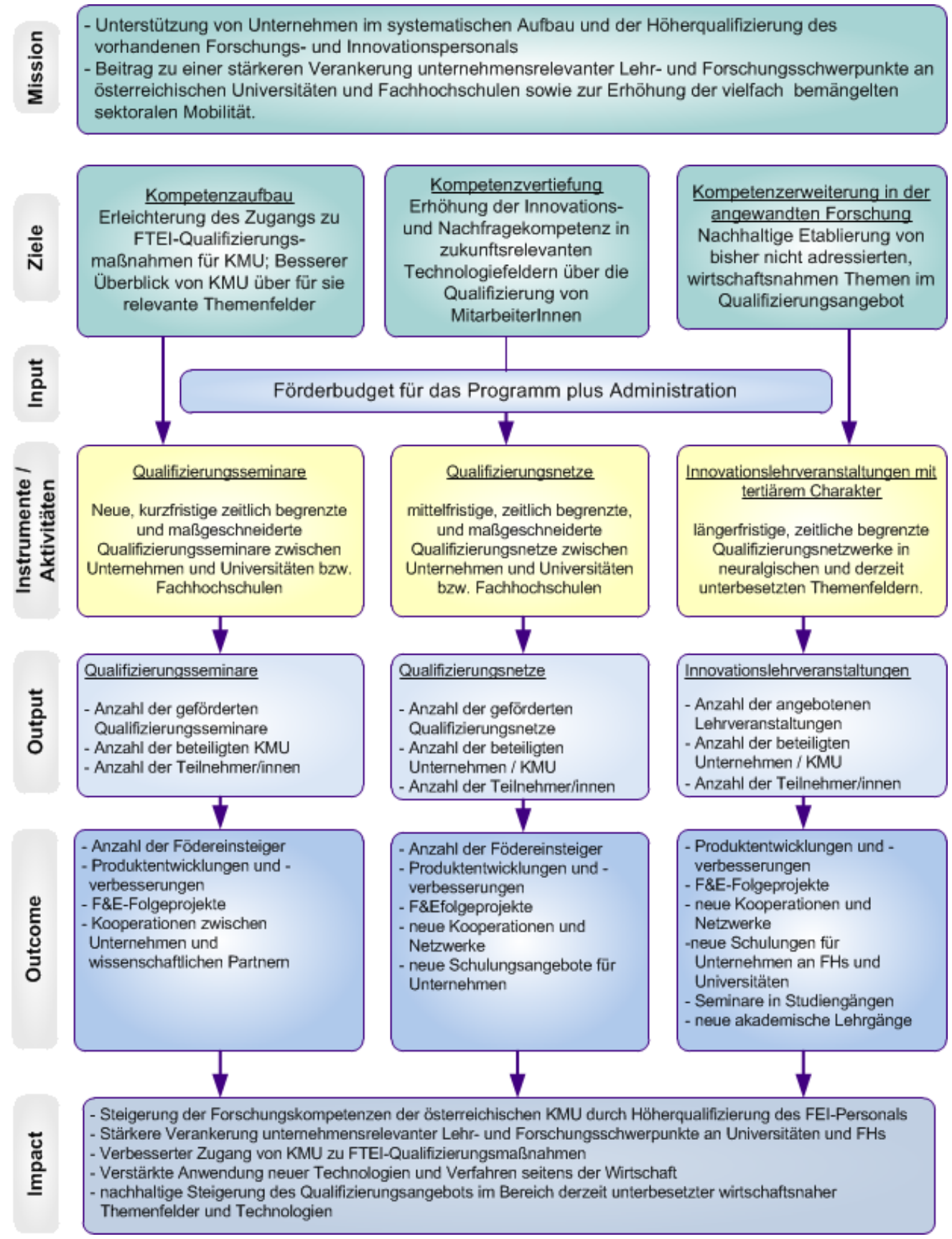
Die operativen Ziele sind den drei Bereichen Kompetenzaufbau, Kompetenzvertiefung und Kompetenzerweiterung sowie den drei Instrumenten des Förderschwerpunkts zugeordnet.

- Kompetenzaufbau: Die formulierten Ziele scheinen in Hinblick auf die Zielgruppe der FTEI-Einsteiger relevant. Von der Konzeption her sind die Qualifizierungsseminare als kurzfristiges, kleines Förderinstrument der Zielgruppe angemessen und können einen erleichterten Zugang von KMU zu Qualifizierungsmaßnahmen im FTEI-Bereich und damit zu relevanten Themenfeldern bieten.

- Kompetenzvertiefung: Die Kompetenzvertiefung adressiert eine breitere Zielgruppe, die neben FTEI-Einsteigern auch technologisch kompetente Unternehmen umfasst. Es wird eine vertiefende Auseinandersetzung mit relevanten Themen angestrebt, die mit dem breiter aufgestellten Instrument der Qualifizierungsnetze, in dessen Rahmen Wissen vertiefend aufgebaut werden soll, erreicht werden kann. Das Ziel der Erhöhung der Innovations- und Nachfragekompetenz in zukunftsrelevanten Technologiefeldern ist aber in diesem Zusammenhang sehr vage formuliert und könnte eher als strategisches Ziel über allen Instrumenten stehen. Andere operative Zielsetzungen wären z.B. der Zugang zu vertiefenden Qualifizierungsmaßnahmen in zukunftsrelevanten Technologiefeldern und die Förderung von nachhaltigen Kooperationen.

- Kompetenzerweiterung: Diese richtet sich an technologisch kompetente Unternehmen und forschungsorientierte Unternehmen und soll zu einer nachhaltigen Etablierung von bisher nicht adressierten, wirtschaftsnahen Themen im Qualifizierungsangebot führen. Mit dieser Zielsetzung werden tertiäre Bildungseinrichtungen adressiert. Die Innovationslehrveranstaltungen mit tertiärem Charakter als längerfristige Qualifizierungsnetzwerke, die auf Hochtechnologiefelder fokussieren sollen, sind das korrespondierende Instrument. Das Instrument der Innovationslehrveranstaltungen war ursprünglich als „industrial PhD-College“ oder als eine Art Stiftungsprofessur geplant, wurde aber im Diskussionsprozess redimensioniert.

Die drei Instrumente stellen aufbauende Instrumente dar: Qualifizierungsseminare als Einsteigerformat, Qualifizierungsnetze als fortgeschrittenes Instrument und die Innovationslehrveranstaltungen mit tertiärem Charakter als Topförderung. Die Instrumente scheinen für die jeweilige Zielgruppe geeignet und den jeweiligen Bedürfnissen angepasst. Dies zeigt sich auch am positiven Feedback der Fördernehmer (siehe Kap. 3.2). Die Innovationslehrveranstaltungen stellen sich als längerfristige Qualifizierungsnetze mit einer fortgeschrittenen Zielgruppe dar. Der Unterschied zu den Qualifizierungsnetzen ist neben der Zielsetzung der „tertiäre Charakter", der Qualifizierungen auf universitärem Niveau hervorbringen soll. Dennoch scheint die Abgrenzung zwischen Qualifizierungsnetzen und Innovationslehr- 
veranstaltungen nicht ganz klar und weist auch laut FFG „Unschärfen“ auf. Zudem scheint der Titel Innovationslehrveranstaltung dem langfristigen Charakter der Förderung nicht gerecht zu werden.

Ob eine Abgrenzung der Instrumente vorliegt, wird auch von den Wirkungen der Instrumente abhängen. Während bei den Qualifizierungsseminaren ein Kompetenzaufbau der MitarbeiterInnen erfolgen soll, der sich in der Produktentwicklung niederschlägt und eventuell kleinere Folgeprojekte hervorbringen kann, sollte es in den Qualifizierungsnetzen zu einer Kompetenzerweiterung kommen. Dies kann dann auch zur Entwicklung oder Verbesserung von Produkten führen und zu F\&EFolgeprojekten. Die Qualifizierungsnetze können sich aber auch in nachhaltigen Kooperationen und verstärkten Weiterbildungsangeboten niederschlagen. Bei den Innovationsveranstaltungen steht neben der Kompetenzerweiterung, die in den Unternehmen durch Transferprojekte unterstützt wird, die nachhaltige Verankerung von Schulungsinhalten im Bildungsangebot im Fokus.

Als Indikatoren bieten sich daher z.B. an:

- Qualifizierungsseminare: Anzahl der Fördereinsteiger, Produktentwicklungen/-verbesserungen, F\&E-Folgeprojekte bzw. neue oder intensivierte Kooperationen zwischen Unternehmen und wissenschaftlichen Partnern

- Qualifizierungsnetzwerke: Anzahl der Fördereinsteiger, Produktentwicklungen/-verbesserungen, F\&E-Folgeprojekte, neue Kooperationen und Netzwerke, Anzahl und Qualität der nachhaltig verankerten Schulungsangebote für Unternehmen

- Innovationslehrveranstaltungen: Produktentwicklungen/-verbesserungen, F\&E-Folgeprojekte, neue Kooperationen und Netzwerke, Anzahl und Qualität der nachhaltig verankerten Schulungen für Unternehmer, Wahlfächer/Übungen/Seminare etc. in Studiengängen, die auf Inhalten der Projekte aufbauen, neue akademische Lehrgänge

Die vorgeschlagenen Indikatoren lassen sich z.B. über eine Befragung der Fördernehmer am Ende der Programmlaufzeit erheben und zeigen, ob die im Rahmen von Forschungskompetenzen für die Wirtschaft eingesetzten Instrumente nachhaltige Wirkungen in Hinblick auf Produktentwicklungen, Netzwerke und das Weiterbildungsangebot haben. Sie können daher Hinweise auf den Impact des Programms geben.

Die im Programmdokument genannten Indikatoren sind dagegen teilweise sehr vage formuliert und kaum operationalisierbar (z.B. „Einschätzung der teilnehmenden Unternehmen über für sie relevante Technologiefelder“) bzw. relational („Anzahl der in FTEl-einsteigenden Unternehmen in der Maßnahme in Relation zur Summe der KMU in der Zielgruppe“) und daher schwer zu erheben. Bei einigen Indikatoren ist auch die Aussagekraft in Frage zu stellen („Anzahl der Teilnehmer an der Qualifizierungsmaßnahme in Relation zur MitarbeiterInnenzahl, die in dem 
Themenkomplex arbeitet"). Auch das BMWFW, das FFG-Programmmanagement sowie die FFG-Strategieabteilung beurteilen die Indikatoren als nicht sehr aussagekräftig bzw. schwer erhebbar und unterstreichen die Notwendigkeit, diese zu überdenken. Zudem wurden Indikatoren nur auf Outcome-Ebene formuliert, es fehlen Impact-Indikatoren.

Zusammenfassend scheint das Konzept des Förderschwerpunkts gelungen. Forschungskompetenzen für die Wirtschaft adressiert ein echtes Defizit und ist innovativ im FFG-Kontext, da es strukturelle Wissenstransfermaßnahmen und die Weiterbildung in Netzwerken fördert. Im Fokus der Förderung stehen Personen, d.h. die MitarbeiterInnen der Unternehmen, und nicht ein Forschungsprojekt. Die Forschungsförderung erfolgt über die Qualifizierung und Weiterbildung von im Feld tätigen Personen. Damit werden Unternehmen auf Zukunftsthemen vorbereitet und ihre Fähigkeit, Innovationen aufzunehmen und umzusetzen wird verbessert. Die Sonderstellung innerhalb der FFG-Förderlogik zeigt sich z.B. auch darin, dass abgelehnte Projekte teilweise als Forschungsprojekte zu klassifizieren waren. Diese besondere Charakteristik macht die Stärke des Programms aus. Es ist ein wirtschaftsgetriebenes Programm, was darin deutlich wird, dass die Unternehmen die Inhalte der Weiterbildung (mit)bestimmen sollen.

Es gibt in Österreich kein vergleichbares Programm, obwohl einige Programme auch die Förderung von Humanressourcen im Fokus haben. So gibt es im Rahmen von COMET sowie im Impulsprogramm "Laura Bassi Centres of Expertise" einen starken Humanressourcenaspekt - beide Programme sind aber Forschungsförderungsprogramme im engeren Sinn und zielen auf exzellente Forschung. Neben Forschungskompetenzen für die Wirtschaft gibt es noch den Förderschwerpunkt Talente, der alle Aktivitäten des BMVIT zur Förderung der Humanpotenziale bündelt. Dieser ist aber mehr nachwuchs- und personenzentriert und fördert v.a. den Einstieg in die Forschung bzw. neues Personal. Auch im europäischen Kontext konnte kein Programm ähnlichen Zuschnitts im engeren Sinn gefunden werden. ${ }^{4}$

Forschungskompetenzen für die Wirtschaft schließt eine Lücke im Förderportfolio der FFG und kann in der Folge auch die Forschungsförderung im engeren Sinn unterstützen, da die Steigerung der Innovationskompetenz in Unternehmen die Qualität der Anträge steigern kann und die Anzahl potenzieller Förderwerber erhöht. Zudem unterstützt der Förderschwerpunkt über die Förderung der Qualifizierung der MitarbeiterInnen die Kompetenzsteigerung in Unternehmen und damit in der Folge den Wirtschaftsstandort - ein Hauptanliegen des Programmeigners BMWFW.

4 Die im Rahmen einer Studie von Technopolis (2008) identifizierten Programme zum Humanressourcenaufbau sind nicht direkt vergleichbar, da die Förderung an die Bearbeitung von Forschungsprojekten im Unternehmen geknüpft ist bzw. neues Personal im Fokus steht und nicht die systematische Weiterbildung bestehenden F\&E-Personals in Netzwerken. 


\section{Die Programmentwicklung}

\subsection{Gesamtübersicht}

Im Laufe der bisherigen Programmabwicklung von Forschungskompetenzen für die Wirtschaft wurden insgesamt 62 Fortbildungsprojekte gefördert. Die angebotenen Qualifizierungsseminare, Qualifizierungsnetze und Innovationslehrveranstaltungen nahmen 553 Unternehmen und Forschungseinrichtungen in Anspruch, wobei einige in mehreren Projekten vertreten waren. Insgesamt liegt die Zahl der Teilnahmen an den drei Programmlinien daher bei 684 (siehe Grafik 2). Mehr als die Hälfte der Projekte und Projektteilnahmen ( $r d .56 \%$ ) konnten bereits abgeschlossen werden (Stand 31.10.2014), 27 Projekte laufen zurzeit. Die Teilnehmerzahl teilt sich auf 448 Unternehmen und 105 Forschungseinrichtungen auf. Letztere umfassen Universitäten (38\%), außeruniversitäre Einrichtungen (20\%), Sonstige (13\%), Fachhochschulen (11\%), Zentren, Cluster und Netzwerke (10\%) sowie Kompetenzzentren (8\%).

\section{Grafik 2 Projekt- und Teilnehmerzahlen (teilnehmende Organisationen)}

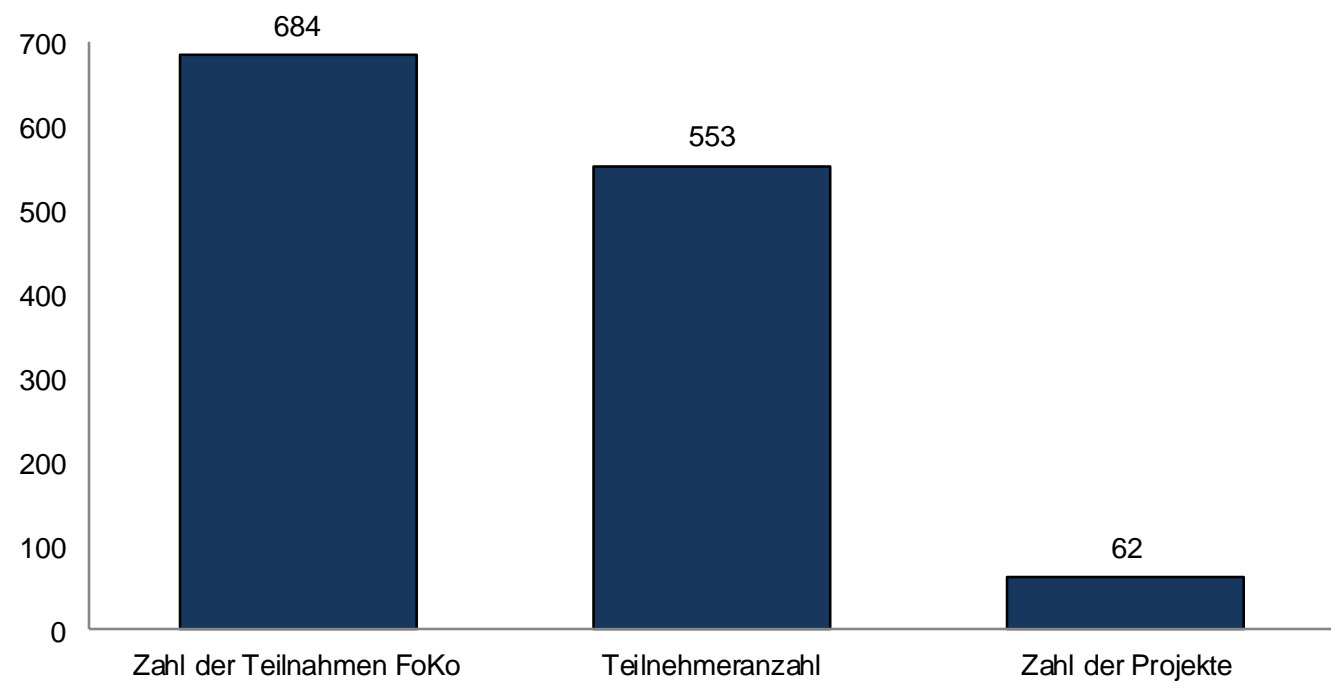

Quelle: eigene Darstellung, Daten FFG Monitoringdaten

Von den 62 Projekten sind 21 (24\%) im Themenfeld IKT angesiedelt und jeweils weitere 15 (24\%) in den Bereichen Energie und Umwelt sowie Produktion. Desweiteren wurden Fortbildungsangebote in den Life Sciences (drei Projekte bzw. $11 \%$ ) und zum Thema Mobilität (ein Projekt bzw. $2 \%$ ) genehmigt. Die übrigen sieben Projekte (11\%) sind unter der Kategorie „Sonstige“ zusammengefasst. 
In Hinblick auf die regionale Verteilung der Fördernehmer zeigt sich eine Konzentration der Fördernehmer in der Steiermark, Niederösterreich, Oberösterreich und Wien (siehe Grafik 3). Aus diesen vier Bundesländern stammen 84 \% der geförderten Unternehmen und Forschungseinrichtungen, wobei mehr als die Hälfte aller Programmteilnehmer in der Steiermark und Niederösterreich ansässig sind. Neben der österreichischen Beteiligung hat auch jeweils ein Projektteilnehmer aus der Schweiz und aus Deutschland (ohne Förderung) teilgenommen.

\section{Grafik 3 Regionale Verteilung der Fördernehmer}

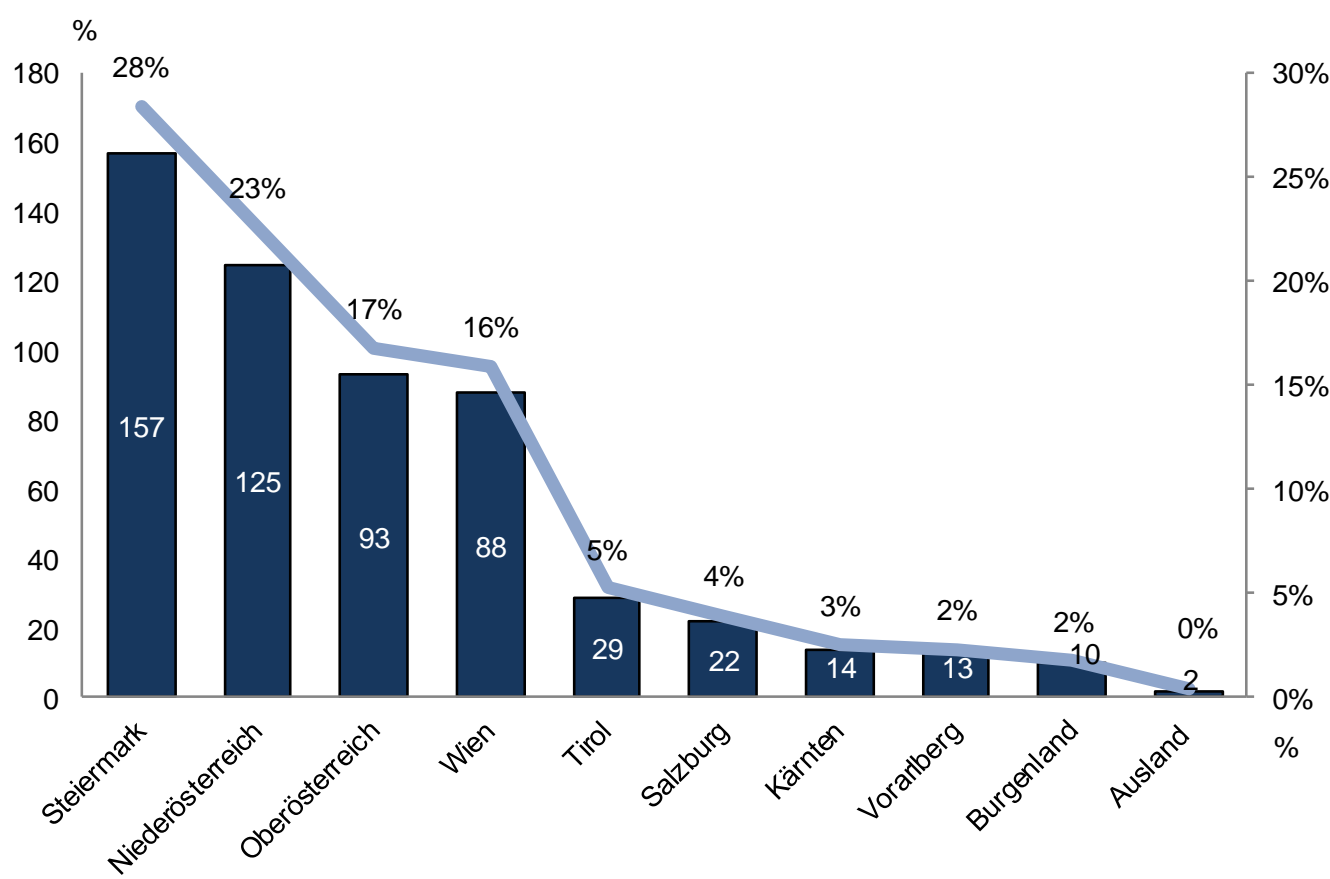

Quelle: eigene Darstellung, Daten FFG Monitoringdaten

Zusammengefasst nach dem primären SIC (Standard Industrial Classification) der Unternehmen und Forschungseinrichtungen zeigt sich, dass rd. $15 \%$ der Fördernehmer im Bereich der Werkstofftechnik tätig sind. Weitere $12 \%$ bzw. $10 \%$ entfallen auf die Bereiche Energieeinsparung, IKT-Anwendungen und Bautechnik. Etwa ein Viertel der Fördernehmer ist in der Kategorie Sonstige zusammengefasst, die u.a. die Bereiche Technologietransfers, Telekommunikation, Wirtschaftsdienstleistungen oder Medizin und Gesundheit beinhaltet (siehe Grafik 4). 


\section{Grafik 4 Branchenverteilung der Fördernehmer, Anteil in \%}

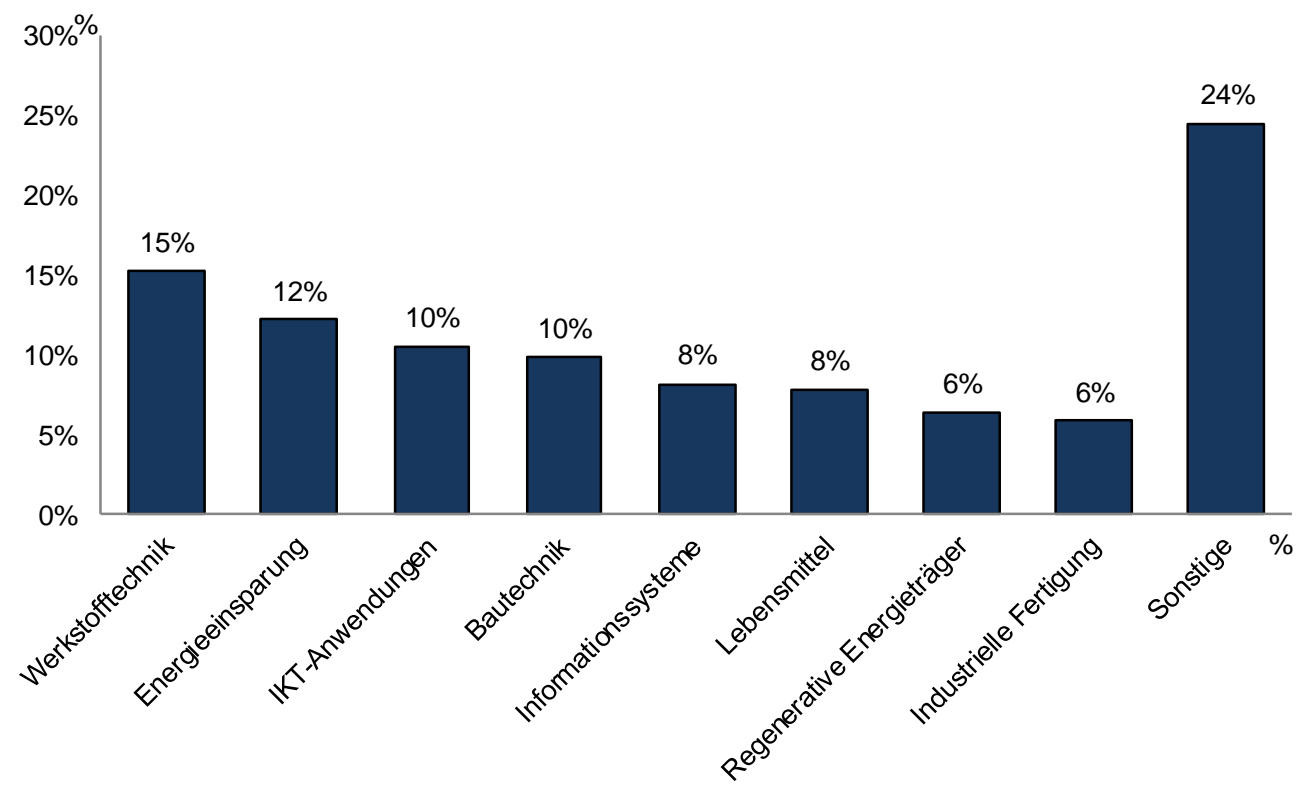

Quelle: eigene Darstellung, Daten FFG Monitoringdaten

Aus den Daten der FFG ist zudem ersichtlich, dass sich einige der Fördernehmer an einer Programmlinie mehrfach beteiligt haben bzw. auch weitere Angebote des Förderschwerpunkts in Anspruch genommen haben. So beteiligten sich drei der untersuchten Forschungseinrichtungen in allen der angebotenen Programmlinien (QS, QN, Inno-LV). Sowohl in Qualifizierungsseminare als auch in Qualifizierungsnetze haben bisher 23 Fördernehmer partizipiert. Davon sind rd. 57 \% Unternehmen bzw. $43 \%$ Forschungseinrichtungen. Die Kombination von Qualifizierungsseminar und Innovationslehrveranstaltung haben vier Fördernehmer in Anspruch genommen (jeweils zwei Unternehmen und Forschungseinrichtungen). Acht Unternehmen haben sich sowohl in den Qualifizierungsnetzen als auch in einer Innovationslehrveranstaltung fortgebildet.

24 Teilnehmer haben mehrfach, dafür aber ausschließlich, an den Qualifikationsseminaren teilgenommen. Davon gehören rd. $70 \%$ dem Unternehmensbereich an und $30 \%$ zu den Forschungseinrichtungen. 27 der untersuchten Fördernehmer haben die Qualifizierungsnetze mehrfach in Anspruch genommen, ohne auf eine weitere Programmlinie zurückzugreifen. Davon waren drei Viertel (20) Unternehmen bzw. $26 \%$ Forschungseinrichtungen (7). Eine Forschungseinrichtung hat an zwei der drei Innovationslehrveranstaltungen partizipiert (siehe Grafik 5). 
Grafik 5 Mehrfache Teilnahmen in Forschungskompetenzen für die Wirtschaft, Anteil in \%

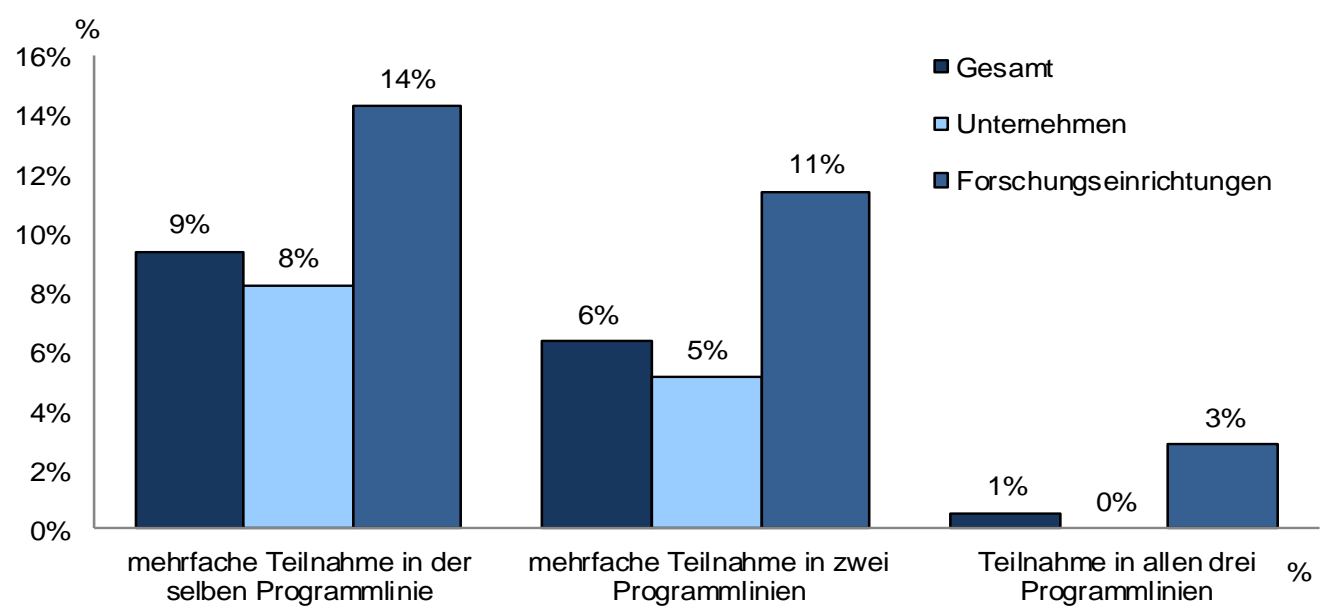

Quelle: eigene Darstellung, Daten FFG Monitoringdaten

Für einen großen Teil der Fördernehmer stellte die Beteiligung an Forschungskompetenzen für die Wirtschaft die erste Unterstützung durch die FFG dar. Insgesamt fanden 189 Unternehmen (rd. $42 \%$ ) und 19 Forschungseinrichtungen (rd. $18 \%$ ) den Zugang zum Förderportfolio der FFG über das Programm (siehe Grafik 6). Die höchste Zahl der Neueinsteiger findet sich mit 107 Fördernehmern bei den Qualifizierungsseminaren. 95 gibt es bei den Qualifizierungsnetzen und sechs bei den Innovationslehrveranstaltungen. Bei den Qualifizierungsseminaren waren die Hälfte der Unternehmen „FFG-Neulinge“, bei den Qualifizierungsnetzen rd. ein Drittel (36\%) und bei den Innovationslehrveranstaltungen knapp jeder Fünfte (17\%).

Grafik 6 Erstfördernehmer in den drei Programmlinien, Anteil in \%

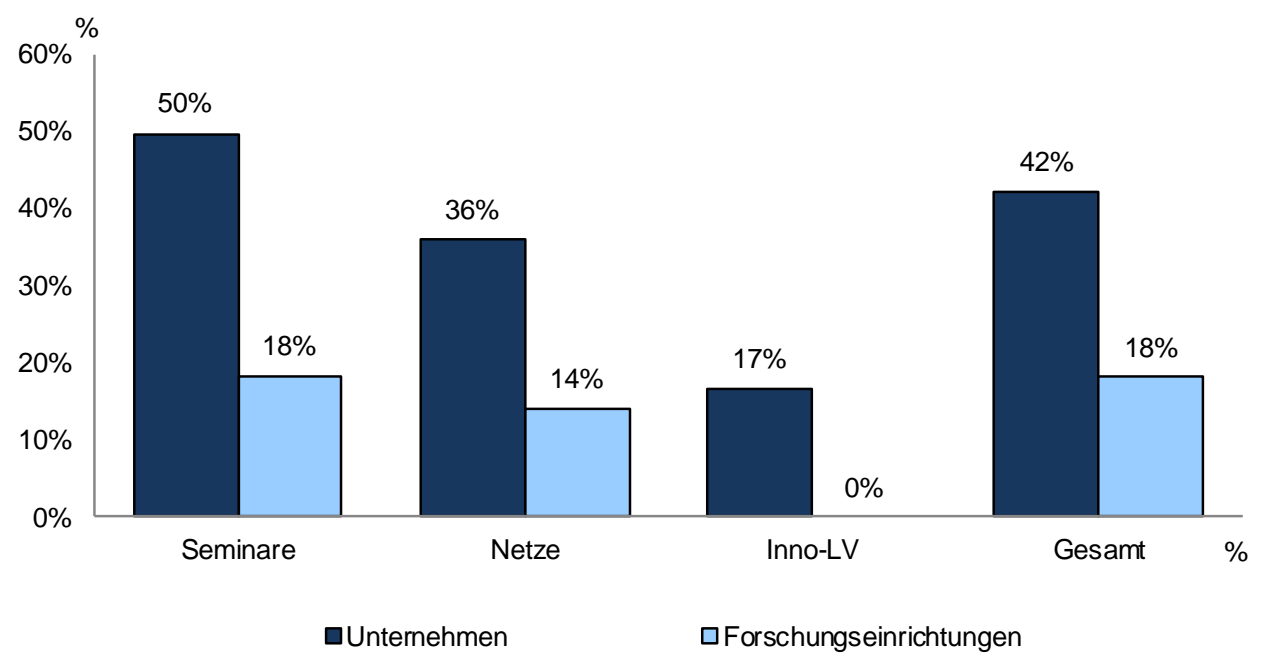

Quelle: eigene Darstellung, Daten FFG Monitoringdaten 
Die Erstfördernehmer zeichneten in weiterer Folge für 43 weitere Beteiligungen im Förderangebot der FFG verantwortlich. Der größte Teil (44\%) davon fällt wiederum auf die unterschiedlichen Programmlinien von Forschungskompetenzen für die Wirtschaft zurück. Weitere Teilnahmen sind in den Programmen Talente (19\%), Innovationsscheck (12\%), COIN (7\%), Basisprogramme und COMET (jeweils $5 \%$ ) sowie Energieforschung (e!Mission), IKT der Zukunft, KIRAS, und Produktion der Zukunft feststellbar (eine Teilnahme bzw. $2 \%$ ).

Wirft man einen Blick auf die Folgeaktivitäten der geförderten Unternehmen nach Durchführung der Projekte im Rahmen von Forschungskompetenzen für die Wirtschaft, so zeichnet sich folgendes Bild: Insgesamt wurden von 152 Fördernehmern weitere 430 FFG Beteiligungen ab dem 1.9.2013 absolviert (davon ausgenommen ist die Beteiligung an Forschungskompetenzen für die Wirtschaft). Der größte Anteil (rd. $23 \%$ ) wurde im Bereich der Basisprogramme abgewickelt, gefolgt von COMET (18\%) und Talente (17\%). Weitere Förderschienen, die vermehrt in Anspruch genommen wurden, sind Energieforschung (e!Mission) (7\%), der Innovationsscheck (6\%) und IKT der Zukunft (5\%). Das verbliebene Viertel der Förderteilnahmen teilt sich auf 17 weitere Programmlinien auf.

Die Differenzierung zwischen Qualifizierungsseminaren und Qualifizierungsnetzen zeigt, dass die Teilnehmerlnnen letzterer häufiger die Angebote der FFG genutzt haben. So haben 82 Unternehmen (rd. 34 \%) insgesamt 301 (rd. $70 \%$ ) der weitere Förderungen ab dem 1.9.2013 beansprucht. Konsequenterweise entspricht die Verteilung auf die Förderfälle nahezu jener der Gesamtverteilung. Wiederum wurden die Basisprogramme am häufigsten in Anspruch genommen (23\%), gefolgt von Talente (19\%) und COMET (16\%). Jeweils rd. $6 \%$ der Förderungen wurden in den Programmen Energieforschung, IKT der Zukunft und Innovationsscheck genützt.

Ein detaillierter Blick auf die Partizipation der Erstfördernehmer zeigt, dass 32 Unternehmen in weiterer Folge für 41 weitere Beteiligungen am Förderangebot der FFG verantwortlich sind (hierbei wurden sämtliche Projekte seit Beginn von Forschungskompetenzen für die Wirtschaft betrachtet). Der größte Teil (46\%) davon fällt wiederum auf die Programmlinien des Förderschwerpunkts zurück. Weitere Teilnahmen sind in den Programmen Talente (19\%), Innovationsscheck (12\%), COIN (7\%), COMET (jeweils $5 \%$ ) sowie Basisprogramm, Energieforschung (e!Mission), IKT der Zukunft und KIRAS (eine Teilnahme bzw. $2 \%$ ). 


\subsection{Die einzelnen Instrumente}

\subsubsection{Qualifizierungsseminare}

Die Qualifizierungsseminare (QS) können als Einsteigerinstrument bezeichnet werden und sollen österreichischen KMU den Zugang zu FTEI-Qualifizierungsmaßnahmen erleichtern und diesen einen besseren Überblick über relevante Technologiefelder bieten. Es handelt sich um kurze, zeitlich begrenzte Qualifizierungsmaßnahmen, die von Universitäten bzw. Fachhochschulen für die teilnehmenden Unternehmen maßgeschneidert konzipiert werden sollen. Zielgruppe sind FTEl-Einsteiger, d. h. Unternehmen mit eher geringer technologischer Kompetenz, die aber die Notwendigkeit des Kompetenzaufbaus und der Innovation im Unternehmen wahrnehmen.

Tabelle 1 Eckpunkte der Qualifizierungsseminare

\begin{tabular}{|l|l|}
\hline Qualifizierungsseminare & Kurzbeschreibung \\
\hline Ziele & $\begin{array}{l}\text { Erleichterung des Zugangs zu FTEI-Qualifizierungsmaß- } \\
\text { nahmen für österr. KMU } \\
\text { Besseren Überblick von KMU über für sie relevante Techno- } \\
\text { logiefelder }\end{array}$ \\
\hline Zielgruppen & $\begin{array}{l}\text { FTEl-Einsteiger (Unternehmen mit geringer technologischer } \\
\text { Kompetenz, Kompetenz- und Innovationsbedarf wird aber } \\
\text { wahrgenommen) }\end{array}$ \\
\hline Inhalt & $\begin{array}{l}\text { Kurzfristige, zeitlich begrenzte und maßgeschneiderte Quali- } \\
\text { fizierungsmaßnahmen für Unternehmen in Kooperation mit } \\
\text { Universitäten und/oder Fachhochschulen werden gefördert. }\end{array}$ \\
\hline Konsortium & $\begin{array}{l}\text { 1. AS: mind. 2 Unternehmenspartner und 1 wissenschaftli- } \\
\text { cher Partner } \\
\text { 2. AS: mind. 2 KMU und 1 Universität/Fachhochschule }\end{array}$ \\
\hline Einreichverfahren & $\begin{array}{l}\text { 2 Ausschreibungen (Juli 2011 - August 2013; Oktober 2013 } \\
- \text { Juni 2014) }\end{array}$ \\
\hline Laufzeit & Max. 6 Monate \\
\hline Förderhöhe & Max $€$ 50.000,- \\
\hline
\end{tabular}

In den ersten beiden Ausschreibungsrunden wurden 38 Qualifizierungsseminare gefördert. 25 davon fielen in die erste Ausschreibung und wurden bereits abgeschlossen, 13 Seminare werden im Rahmen der zweiten Ausschreibung gefördert und zum Zeitpunkt der Berichterstellung durchgeführt. Im Rahmen der ersten Ausschreibung wurden insgesamt 34 Qualifizierungsseminare zur Förderung eingereicht, im Zuge der zweiten Ausschreibung 18. Damit liegen die Quoten der erfolgreichen Antragstellung jeweils bei über $70 \%$ (74 \% bzw. $72 \%)$. 
Die 38 Qualifizierungsseminare umfassen insgesamt 302 Beteiligungen durch Unternehmen (215) und Forschungseinrichtungen (87). 193 Unternehmen und 60 Forschungseinrichtungen werden hier gefördert. Die Abweichungen dieser Werte lassen sich durch die Mehrfachteilnahmen einiger Fördernehmer erklären und sind in Grafik 7 dargestellt. 16 Unternehmen und neun Forschungseinrichtungen haben an zwei Qualifikationsseminaren teilgenommen bzw. jeweils drei Organisationen waren in drei verschiedenen QS vertreten. Darüber hinaus haben zwei Forschungseinrichtungen an 7 der bisher durchgeführten Seminare teilgenommen.

\section{Grafik 7 Teilnahme der Unternehmen und Forschungseinrichtungen an Qualifi- zierungsseminaren}

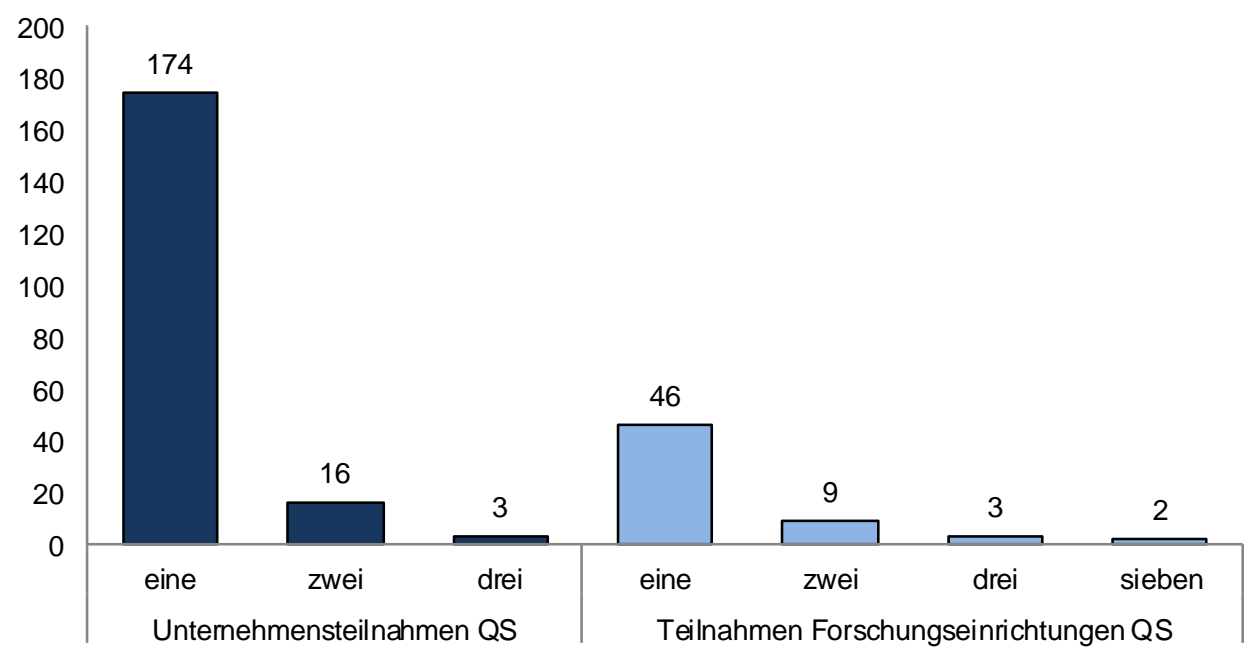

Quelle: eigene Darstellung, Daten: FFG Monitoringdaten

Anmerkung: Hier wird nur die Förderschiene Qualifizierungsseminare betrachtet. Einige der Organisationen wurden auch im Rahmen der Qualifizierungsnetze oder der Innovationslehrveranstaltungen gefördert. Dies wird in Kap. 3.1 diskutiert.

In zumindest zwei Fällen lassen sich Projektketten erkennen, d. h. zwei Konsortien haben in nahezu gleichbleibender Konstellation zwei bzw. drei Qualifizierungsseminare mit aufbauendem Inhalt durchgeführt. Darüber hinaus kam es in jeweils einem Fall zu einer teilweisen Fortsetzung von Konsortialstrukturen in einer Innovationslehrveranstaltung bzw. in einem weiteren Qualifikationsseminar. 
In einem Qualifizierungsseminar sind durchschnittlich 5,7 Unternehmen und 2,3 Forschungseinrichtungen konzentriert. Das umfangreichste Einzelprojekt weist 22 Teilnehmer auf, wovon 19 Unternehmen und drei Forschungseinrichtungen sind. Demgegenüber stehen zwei Seminare, die lediglich drei Teilnehmer aufweisen (jeweils zwei Unternehmen und eine Forschungseinrichtung). In 30 der 38 Qualifizierungsseminare waren die Forschungseinrichtungen Konsortialführer, wobei außeruniversitäre Einrichtungen (9) und Fachhochschulen (8) am häufigsten die Projekte geleitet haben. Von den 8 Projekten unter Unternehmensleitung wurden 6 von Kleinunternehmen durchgeführt. Mittlere und Großunternehmen waren jeweils einmal Konsortialführer.

Thematisch ist der größte Anteil der Projekte im Bereich IKT angesiedelt (42\%). Auf die Bereiche Energie und Umwelt sowie Produktion entfallen jeweils 7 (18\%) der Projekte. Darüber hinaus werden auch die Themenfelder Life Science und Mobilität (jeweils ein Projekt) bedient. Die verbliebenen Projekte (6) wurden nicht näher thematisch zugeordnet.

Bezogen auf die regionale Verteilung ist der größte Anteil der Fördernehmer aus dem Unternehmensbereich in der Steiermark (30\%) zu finden (siehe Grafik 8). Weniger als ein Fünftel der Unternehmen stammen jeweils aus Wien (18\%), Nieder- $(17 \%)$ und Oberösterreich (17\%). Die größte Zahl der Forschungseinrichtungen ist hingegen in Wien angesiedelt (30\%) gefolgt von der Steiermark (28\%) und Oberösterreich (22\%).

\section{Grafik 8 Regionale Verteilung der Fördernehmer der Qualifizierungsseminare}

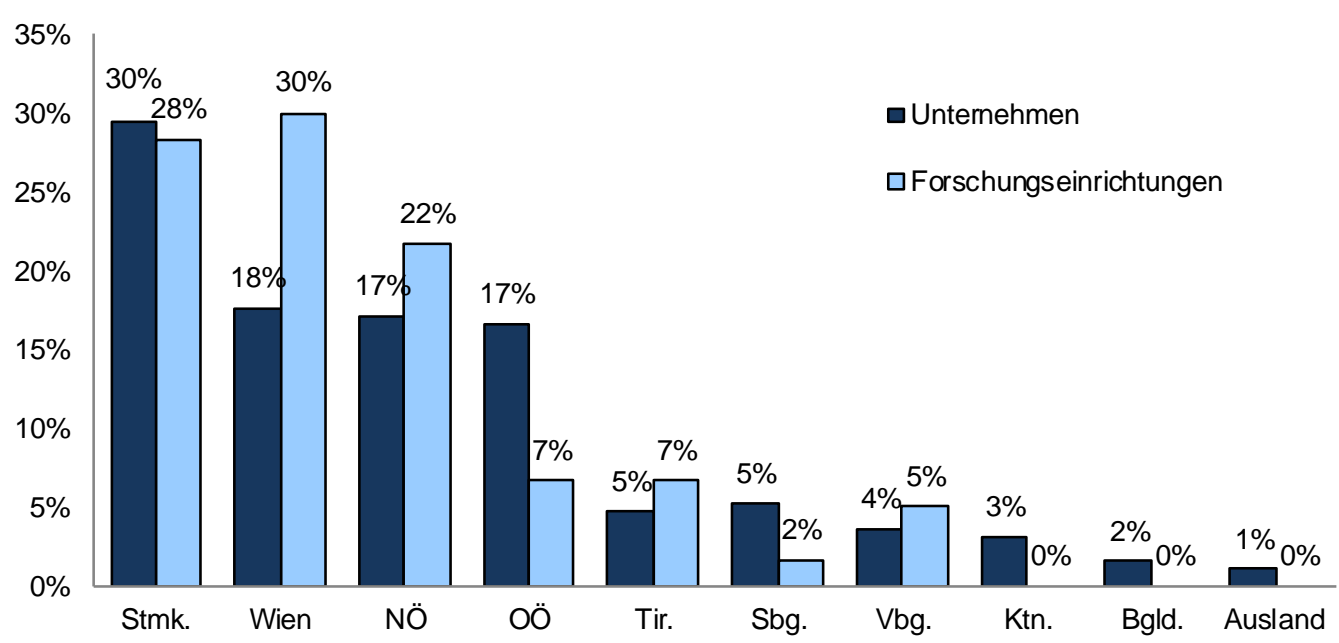

Quelle: eigene Darstellung, Daten: FFG Monitoringdaten 
Des Weiteren lassen sich die teilnehmenden Unternehmen bezüglich Ihrer GröBenklasse klassifizieren. Mehr als drei Viertel der Unternehmen weisen den Status eines Kleinunternehmens auf $(77 \%)$ und weitere $16 \%$ können als mittlere Unternehmen charakterisiert werden. Großunternehmen (7\%) nehmen kaum an Qualifizierungsseminaren teil.

Für 107 der 253 Teilnehmer der Qualifizierungsseminare (rd. $42 \%$ ) stellt die Programmlinie eine Erstförderung durch die FFG dar. Der überwiegende Anteil (96) der Erstfördernehmer sind Unternehmen und davon $92 \%$ Kleinunternehmen. Dementsprechend ist etwa jedes zweite Unternehmen ( $r d .50 \%$ ) ein Neukunde der FFG. ${ }^{5}$ Bei den Forschungseinrichtungen sind hingegen nur $18 \%$ erstmalige Fördernehmer, wobei es sich u.a. um außeruniversitäre Forschungseinrichtungen, Intermediäre (Cluster) und Einzelforscher handelt.

\section{Ziele und Motivation}

Ziel der Seminare war in den meisten Fällen die Vermittlung anwendbaren Wissens und die Kompetenzerweiterung der beteiligten Unternehmen in einem spezifischen Themenbereich. Dies steht im Einklang mit den Ausschreibungsleitfäden, in denen spezifiziert wird, dass im Mittelpunkt der Förderung Qualifizierungsmaßnahmen für den Einstieg in FTEl-Themen stehen sollen, damit in der Folge Anzahl und Volumen der FTEl-Aktivitäten gesteigert werden. Die Motivation zur Teilnahme war denn auch bei den Unternehmen in erster Linie inhaltsgetrieben. Das Thema wurde meist schon im Vorfeld als wichtig für die Weiterentwicklung des Unternehmens erkannt sowie Weiterbildungsbedarf in diesem Bereich identifiziert. So befassten sich z. B. einige Seminare mit den technischen Voraussetzungen der Einführung von ELGA oder Grundlagen und neuen Methoden für die Entwicklung von Apps. Aber auch Grundlagenwissen in bestimmten Bereichen, die Voraussetzung zur Weiterentwicklung von Produkten sind, wurde vermittelt. Dabei handelte es sich zumeist um zumindest teilweise neue Technologiefelder und Themen, die bis dato kein Schwerpunkt im Unternehmen waren, wobei eine Vertiefung aber angedacht war. Rd. ein Drittel der Qualifizierungsseminare der 1. Ausschreibung hatte einen methodischen Schwerpunkt: Hier sollten den Unternehmen in erster Linie methodische Kompetenzen (z.B. „systemisches Innovieren“, „Innovationsmanagement") vermittelt werden, die in der Unternehmensorganisation verankert werden können, um Innovationen unabhängig vom Thema voranzutreiben.

5 Ein ähnlicher Wert (rd. 52 \%) lässt sich für die geförderten Unternehmen der Förderschiene Innovationsscheck Plus feststellen, welche ebenfalls als Einstiegsinstrument zur unternehmerischen Innovationstätigkeit fungiert. 
Dass es bis dato zu keiner Weiterbildung in den als relevant erkannten Bereichen gekommen war, erklärten die Unternehmen mit einem mangelnden Angebot vor Ort und der mangelnden Zeit für die Planung im betrieblichen Alltag. Das Qualifizierungsseminar wurde daher als perfekte Gelegenheit gesehen, hier Qualifizierungsdefizite abzubauen. Ziel der Teilnahme war es denn auch, MitarbeiterInnen, die sich im Unternehmen mit der Thematik auseinandersetzen, in diesem Bereich zu qualifizieren und direkt anwendbares Wissen zu generieren.

Die Förderung der Personalkosten gab für einige Unternehmen nicht den Ausschlag für die Teilnahme am Qualifizierungsseminar. Einige nannten diese als Goodie, andere betonten aber, dass sie die Weiterbildungsmaßnahme nicht in diesem Umfang durchgeführt hätten („Die Entscheidung wäre uns nicht so leicht gefallen."). Zudem wurde betont, dass normalerweise solche Weiterbildungen neben den Personalkosten mit teilweise erheblichen Schulungskosten verbunden sind, sodass diese nur seltener und mit weniger Teilnehmerlnnen in Anspruch genommen werden können.

\section{Ausgestaltung}

Der Zugang zum Qualifizierungsseminar erfolgte auf unterschiedlichen Wegen. Einige interviewte Unternehmen wurden von den wissenschaftlichen Partnern angesprochen, wobei hier teilweise schon Kooperationen im Vorfeld bestanden. Andere wurden von anderen Unternehmen zur Teilnahme aufgefordert oder hörten über den Cluster, bei dem sie Mitglied sind, über das Projekt.

Die Detailplanung der Schulungen erfolgte zum Großteil in Abstimmung mit den Unternehmen (in meist bilateralen Projektmeetings und per E-Mail), um dem konkreten Qualifizierungsbedarf begegnen und auf die Bedürfnisse der Unternehmen eingehen zu können. In einigen Seminaren wurden kleinere Adaptierungen auch noch während der Seminarphasen auf Basis der Feedbacks der Teilnehmerlnnen vorgenommen.

An den Seminaren selbst nahmen 374 (1. Ausschreibung) Personen teil bzw. werden laut Planung weitere 142 Personen (Stand Oktober 2014) teilnehmen (2. Ausschreibung). Etwa 14\% der Seminarteilnehmerlnnen (1. Ausschreibung) waren weiblich, in der zweiten Ausschreibung liegt der geplante Anteil derzeit bei etwa $25 \%$. Allerdings schwankt die „Frauenquote" bei den einzelnen Seminaren beträchtlich (Grafik 9 und Grafik 10). In 9 Seminaren (2. Ausschreibung: zwei Seminare) gab es keine einzige Teilnehmerin, während 6 Seminare relativ hohe Frauenquoten (27\% bis $50 \%$ ) von über einem Viertel aufweisen (2. Ausschreibung fünf Projekte zwischen $30 \%$ und $63 \%$ ). 
Grafik 9 Teilnehmerlnnenanzahl und Frauenanteil der Qualifizierungsseminare der ersten Ausschreibung

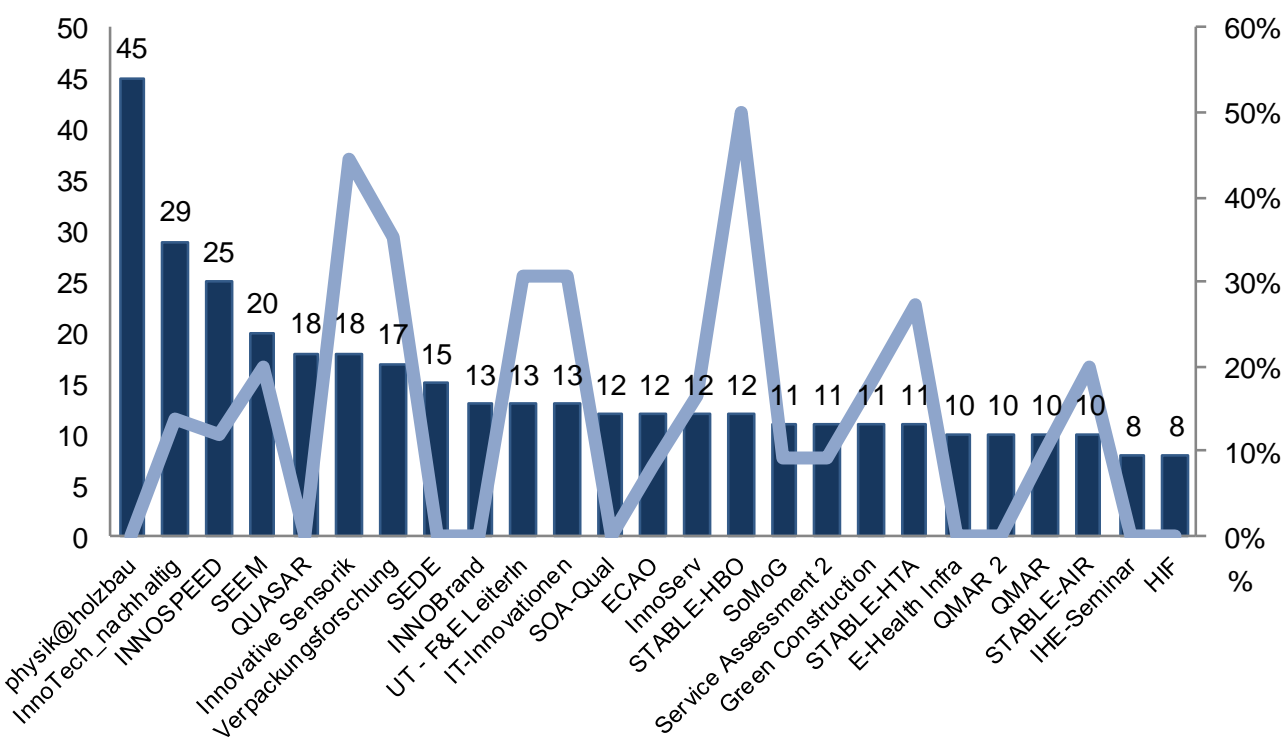

Quelle: eigene Darstellung, Daten: interne Auswertung FFG

Grafik 10 Vorläufige Teilnehmerlnnenanzahl und Frauenanteil der Qualifizierungsseminare der zweiten Ausschreibung

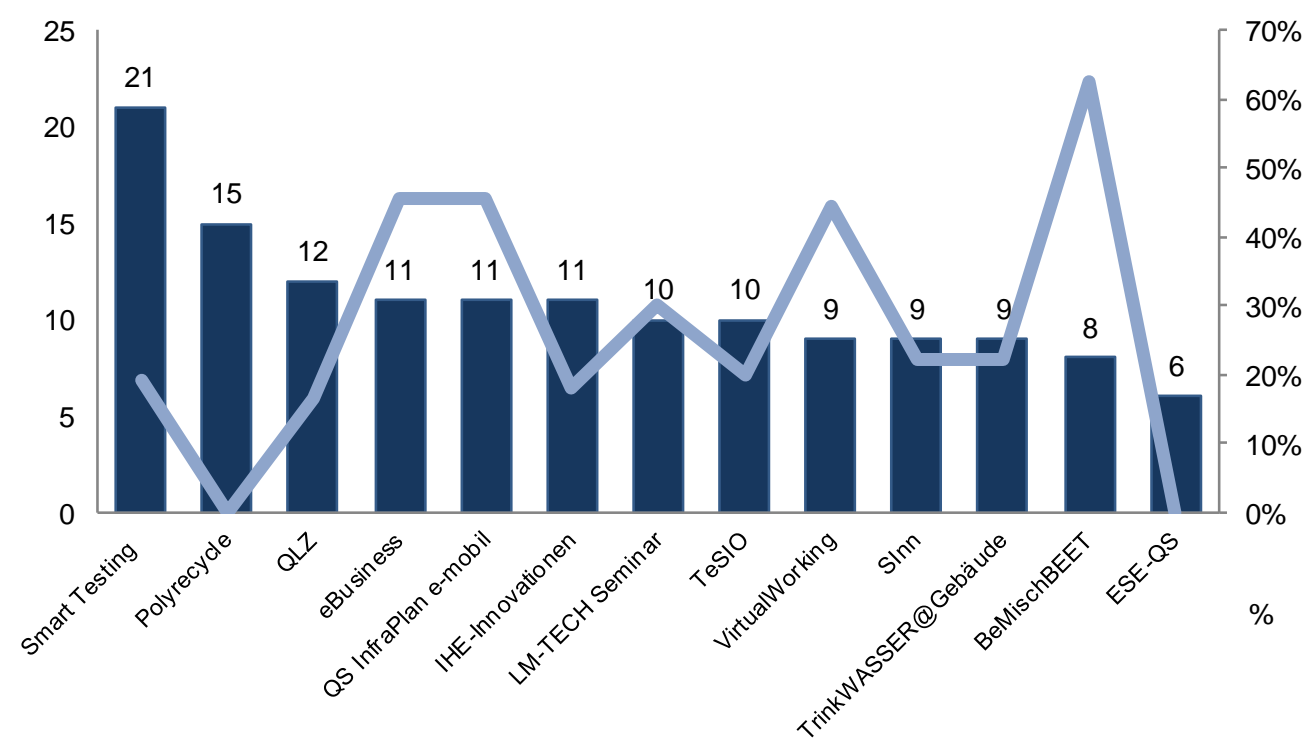

Quelle: eigene Darstellung, Daten: interne Auswertung FFG 
Obwohl laut Ausschreibungsunterlagen bei der Zusammensetzung der TeilnehmerInnen auf ein ausgewogenes Geschlechterverhältnis zu achten ist, gelingt dies in den wenigsten Fällen. Dies spiegelt die Unterrepräsentanz von Frauen in technologischen Branchen im Allgemeinen sowie die Teamzusammensetzung in den einzelnen Unternehmen wider, da im Rahmen der Förderung bestehendes Personal geschult und nicht neues aufgebaut werden soll. In den Interviews wird denn auch deutlich, dass dort, wo mehr Frauen in relevanten Bereichen tätig sind, diese auch die Seminare besuchten („Wir haben die Frauenquote übererfüllt, weil wir mehr Frauen im Team haben. "). Bei anderen Unternehmen finden sich keine Frauen in den relevanten Positionen („Bei uns sind zurzeit keine Frauen im technischen Bereich tätig.").

Die Teilnehmerlnnen der Seminare wurden meist von der Geschäftsführung, manchmal in Absprache mit AbteilungsleiterInnen und unter Berücksichtigung der Interessenslage der MitarbeiterInnen nominiert. Bei den kleinen Unternehmen nahm oftmals auch der/die Geschäftsführerln an den Schulungen teil. Bei manchen Seminaren kam es aufgrund des hohen Interesses auch zu zusätzlichen Teilnahmen von ursprünglich nicht geplanten Personen (ohne Förderung).

Die Seminare selbst beinhalteten meist einen einführenden theoretischen Teil und dann interaktive Elemente, wie z.B. Workshops, Übungen am Gerät, Livepräsentationen, Werkbesichtigungen, Exkursionen, Laborübungen, Übungen an Realbeispielen etc. Einige wenige Seminare richteten auch E-learning Plattformen ein.

Dabei fanden v.a. die praktischen und interaktiven Übungen, bei denen das Gelernte am Gerät demonstriert wurde und von den Teilnehmerlnnen direkt umgesetzt werden konnte, besonderen Anklang. Die Praxisrelevanz der Seminare wurde hervorgehoben. Um diese zu unterstützen, konnten bei einigen Seminaren die Unternehmen firmeninterne Projekte einbringen, die im Laufe des Seminars mithilfe des Erlernten bearbeitet wurden. Das Feedback der TeilnehmerInnen, das meist nach den Schulungen eingeholt wurde (Fragebogen und/oder Feedbackgespräche), war fast durchwegs (sehr) gut. Auch wurden die SeminarleiterInnen und ExpertInnen, die die Schulungen durchführten, als äußerst kompetent und bemüht, auf die Bedürfnisse der TeilnehmerInnen einzugehen, beurteilt.

\section{Kooperationen}

Die Kooperation innerhalb der Konsortien dürfte reibungslos und sehr gut funktionieren. Bei der Konsortialführung laufen die Fäden zusammen, diese sind für die Administration und Organisation der Seminare zuständig. Damit wird der administrative Aufwand für die Unternehmen auf ein Minimum reduziert, was diese sehr schätzen. Besonders für die Fördereinsteiger ist es von Vorteil, wenn mit FFGFörderungen vertraute Organisationen die administrative Abwicklung der Projekte übernehmen. 
Auch die interne Organisation der Schulungen erfolgte zur Zufriedenheit, wenngleich die Terminfindung teilweise als problematisch eingestuft wurde. V.a. bei kleinen Unternehmen war es nicht immer leicht, die Teilnehmerlnnen vom Tagesschäft freizuspielen, sodass diese an den Schulungen teilnehmen konnten. Auch in den Endberichten wird von einigen Konsortialführern erwähnt, dass die Terminkoordination der Schulungen eine Herausforderung darstellte, obwohl die Vortragenden meist große Flexibilität an den Tag legten.

Das Qualifizierungsseminar bedeutete für einige Unternehmen einen ersten Kontakt zu einem wissenschaftlichen Partner, in anderen Fällen wurde die Zusammenarbeit des wissenschaftlichen Partners mit den Unternehmenspartnern gefestigt. Aber auch der Kontakt zu anderen Unternehmen wurde positiv hervorgehoben. Wenn Unternehmen der gleichen Branche in einem Seminar zusammen geschult wurden, kam es zu einem regen Erfahrungsaustausch mit den Mitbewerbern. Vorbehalte, Probleme mit der „Konkurrenz“ zu diskutieren, zeigten sich kaum, eher zeigte man sich erfreut mit "Gleichgesinnten" zusammenzukommen. Bei branchenübergreifenden Seminaren berichteten die Unternehmen von den Vorteilen, andere Sichtweisen kennengelernt zu haben, die es nun auch ermöglichen, den Fokus auf ganzheitliche Lösungen zu legen.

Wenngleich nur fallweise konkrete neue Kooperationen mit anderen teilnehmenden Unternehmen vereinbart wurden, betonen die Interviewten, dass sie es schätzen, nun Kontakt zu Unternehmen zu haben, die z.B. andere Bereiche in der Wertschöpfungskette abdecken. So hätten Sie nun bei bestimmten Problemen neue AnsprechpartnerInnen. Unternehmen, die bereits vor dem Qualifizierungsseminar kooperierten, werden dies auch weiterhin tun. Eine Zusammenarbeit mit den wissenschaftlichen Partnern können sich alle interviewten Unternehmen vorstellen, unabhängig davon, ob schon zuvor Kontakt bestand oder nicht.

\section{Wirkungen}

Die Qualifizierungsseminare erzielen Wirkungen auf die Projektpartner in folgenden Bereichen:

- Kompetenzaufbau und Stärkung der Innovationskompetenz: Aus den Rückmeldungen der Unternehmen geht hervor, dass es durch die Seminarteilnahme in allen Fällen zu einem Know-How Aufbau bei den teilnehmenden Personen gekommen ist. Es wurde zum einen inhaltliches KnowHow bzgl. der in den Seminaren behandelten Themen aufgebaut (,tieferes Verständnis der Thematik“, „sensibilisiert für Probleme“, „Begrifflichkeiten jetzt klar"), zum anderen ein Verständnis für Innovationsprozesse entwickelt. Dies bedeutet für die Unternehmen eine Stärkung ihrer Innovationskompetenz, aber auch z. B. die Möglichkeit, Kundlnnen besser beraten zu können. Vor allem bei branchenübergreifenden Qualifizierungsseminaren 
konnte erreicht werden, dass die Teilnehmerlnnen nunmehr über den eigenen Tellerrand blicken und ganzheitlicher denken. Damit wird auch die Kommunikation mit Kooperationspartnern und Kundlnnen leichter (,jetzt können wir auf einen anderem Level mit unserem Bauphysiker sprechen"). Teilweise konnte mit der Seminarteilnahme auch die Sachverständigentätigkeit auf eine breitere Wissensbasis gestellt werden. Die mehr methodisch ausgerichteten Seminare führten zur Optimierung interner Unternehmensprozesse („Das wurde für uns fixer Bestandteil unserer Unternehmenskultur - wir haben jetzt eine Innovationsgruppe im Unternehmen, die sich regelmäßig trifft."). Aber auch die inhaltlich orientierten Seminare führten zu Veränderung in der methodischen Vorgehensweise bei Entwicklungen („ist Teil der Tagesarbeit geworden"). Dies alles kann zu erheblichen Wettbewerbsvorteilen führen (,jetzt sind wir weniger vergleichbar").

- Produktentwicklung: Infolge der Seminarteilnahme wurde in vielen Unternehmen das neu angeeignete Wissen dazu verwendet, um neue Produkte/Dienstleistungen zu entwickeln bzw. bestehende Produkte/Dienstleistungen zu erweitern oder zu verbessern. Dadurch kam es zu einer Erweiterung des Angebots, womit neue Kundengruppen adressiert werden können („wir gehen mit der neuen Technologie aktiv auf die Kunden zu“). Beispiele hierfür sind der Einbau von Schutzmechanismen in SmartphoneApplikationen (,80 \% der Bedrohungen sind weg"), die Entwicklung von Apps, die Entwicklung einer neuen Verpackungslinie oder die Adaptierung der Konstruktionen auf Basis neuester wissenschaftlicher Erkenntnisse. Einige Unternehmen rüsteten sich mithilfe der Seminarteilnahme für geänderte Rahmenbedingungen (ELGA-Einführung), damit ihre Produkte anschlussfähig werden bzw. sie auch die Kundlnnen entsprechend beraten können („,wir können den Kunden jetzt die Unsicherheit nehmen“). Auch wenn kein konkretes Produkt entwickelt wurde, fließen die Erfahrungen nach eigenen Angaben in die Entwicklung neuer Produkte mit ein bzw. befinden sich Unternehmen in Ideenfindungsprozessen.

- Weiterentwicklung des Qualifizierungsangebots: Einige wissenschaftliche Partner nützen die Erfahrungen aus dem Qualifizierungsangebot für die Lehre. Die Inputs der Seminare lieferten Bausteine für Vorlesungen und Praxiseinheiten sowie inhaltliche Anregungen für Diplom- und Masterarbeiten. In zwei Fällen sind Abschlussarbeiten bzw. studentische Projekte in Partnerunternehmen geplant. In einem Fall wird das Curriculum eines Masterstudiengangs durch die Integration einer Lehrveranstaltung zum Thema des Qualifizierungsseminars adaptiert. Ein Cluster integriert einzelne adaptierte Seminare in das reguläre Weiterbildungsprogramm. Auch werden die Lehrunterlagen teilweise für andere Schulungen weiterverwendet. 
- Folgeprojekte und F\&E-Aktivitäten: In einigen Fällen kam es eigenen Angaben zufolge zu neuen Forschungsprojekten. Von den Unternehmen, die an den Qualifizierungsseminaren partizipiert haben, nutzten 55 (rd. 28\%) weitere FFG Angebote. Die meistgenutzten Förderprogramme waren dabei die Basisprogramme (25\%), COMET (17\%), Innovationsscheck (9\%), Talente (9\%), Energieforschung (7\%) und Technologiekompetenzen (6\%). ${ }^{6}$ Zudem nehmen einige Unternehmen an zwei Qualifizierungsseminaren teil oder neben dem Qualifizierungsseminar an einem Qualifizierungsnetz (siehe Grafik 7 bzw. Kap. 3.1). Die meisten Unternehmen werden ihre F\&E-Aktivitäten auf dem derzeitigen Niveau halten, einige wenige gaben auch an, diese steigern zu wollen.

\section{Beurteilung}

Insgesamt wird die Programmlinie Qualifizierungsseminare als sehr geeignetes Instrument zur Kompetenzsteigerung von KMU gesehen. Dies wird auch darin deutlich, dass sich alle Interviewten eine neuerliche Teilnahme vorstellen können, wenn das Thema für sie von Relevanz ist („Sofort schicke ich wen wieder hin."). Einige Unternehmen sind auch bereits in mehr als einem Qualifizierungsseminar eingebunden (rd. $10 \%$ ). Bei anderen wird die Nachfrage nach einer fortführenden Ausbildung deutlich bzw. weiterer Qualifizierungsbedarf geortet.

Als Erfolgsfaktoren konnten identifiziert werden:

- Die Inhalte werden maßgeschneidert an die Bedürfnisse der Unternehmen angepasst („So eine fokussierte Schulung ist sehr willkommen.").

- Durch die Einbindung der wissenschaftlichen Partner wird ein Einblick in den aktuellsten Stand der Forschung im jeweiligen Bereich geboten (,Nebenbei erfährt man so viel von den wissenschaftlichen Entwicklungen").

- Der Erfahrungsaustausch und die Interaktion mit den anderen Unternehmen bzw. Teilnehmerlnnen wird als echter Mehrwert bezeichnet („Da sind Firmen dabei, die das Thema weiterbringen möchten."). Dies gilt sowohl für Seminare, in denen Unternehmen der gleichen Branche geschult werden, als auch für branchenübergreifende Seminare. In letzterem Fall wird die Einbringung verschiedener Perspektiven, die zu einer ganzheitlichen Betrachtung der Probleme führt, begrüßt („Thema wird von verschiedenen Seiten beleuchtet $\left.{ }^{\prime}\right)$. Der Austausch werde auch durch den kleinen Kreis an Teilnehmerlnnen erleichtert.

${ }^{6}$ Quelle: FFG-Monitoringdaten 
Die Qualifizierungsseminare bieten einen guten Rahmen für Weiterbildungsmaßnahmen in KMU. Zudem wird der Kontakt zur Wissenschaft erleichtert. Damit können zum einen Barrieren abgebaut und KMU der Zugang zu Forschungseinrichtungen erleichtert werden, zum anderen erhalten die Forschungseinrichtungen wichtige Inputs aus der Praxis zur Formulierung neuer Forschungsfragen und zur Gestaltung ihrer Qualifizierungsangebote. Die Qualifizierungsseminare erweisen sich sowohl in ihrer Konzeption als auch in ihrer praktischen Ausgestaltung als Einsteigerformat und sind besonders geeignet für kleine Unternehmen. 


\subsubsection{Qualifizierungsnetze}

Die Qualifizierungsnetze sind mittelfristige Qualifizierungsmaßnahmen für Unternehmen in Kooperation mit Universitäten und Fachhochschulen. Die Projekte richten sich an FTEI-Einsteiger und technologisch kompetente Unternehmen mit eigenem F\&E-Budget. Ziel ist es, die Innovations- und Nachfragekompetenz der teilnehmenden Unternehmen in zukunftsrelevanten Technologiefeldern zu erhöhen.

Tabelle 2 Eckpunkte der Qualifizierungsnetze

\begin{tabular}{|l|l|}
\hline Qualifizierungsnetze & Kurzbeschreibung \\
\hline Ziele & $\begin{array}{l}\text { Erhöhung der Innovations- und Nachfragekompetenz in zu- } \\
\text { kunftsrelevanten Technologiefeldern über die Qualifizierung } \\
\text { von Mitarbeitenden }\end{array}$ \\
\hline Zielgruppen & $\begin{array}{l}\text { FTEl-Einsteiger } \\
\text { Technologisch kompetente Unternehmen (Unternehmen mit } \\
\text { mehreren Ingenieurlnnen bzw. Forscherlnnen und einen } \\
\text { eigenen F\&E-Budget) }\end{array}$ \\
\hline Inhalt & $\begin{array}{l}\text { Es werden mittelfristige, zeitlich begrenzte und maßge- } \\
\text { schneiderte Qualifizierungsmaßnahmen für Unternehmen } \\
\text { (speziell KMU) in Kooperation mit Universitäten und/oder } \\
\text { Fachhochschulen gefördert. }\end{array}$ \\
\hline Konsortium & $\begin{array}{l}\text { 1. Ausschreibung: min. 3 Unternehmenspartner (davon min. } \\
\text { KMU) und 1 wissenschaftlicher Partner } \\
\text { 2. Ausschreibung: mind. 3 voneinander unabhängige KMU } \\
\text { und 1 Universität/Fachhochschule }\end{array}$ \\
\hline Einreichverfahren & $\begin{array}{l}\text { 2 Ausschreibungen (Oktober 2011- März 2012; Oktober 2013 } \\
- \text { März 2014) }\end{array}$ \\
\hline Laufzeit & Min. 6 Monate bis max. 24 Monate \\
\hline Förderhöhe & Max. $€ 500.000,-$ \\
\hline
\end{tabular}

Insgesamt wurden 21 Qualifizierungsnetze (QN) gefördert. Davon wurden $10 \mathrm{im}$ Zuge der ersten Ausschreibung abgewickelt bzw. 11 in der zweiten Ausschreibung. Für eine Förderung eingereicht wurden 20 Projekte (1. Ausschreibung) bzw. 19 Qualifizierungsvorhaben (2. Ausschreibung). Dementsprechend liegt der Anteil erfolgreicher Antragsstellungen bei $50 \%$ bzw. $58 \%$.

In den Qualifizierungsnetzen können bisher 339 Teilnahmen gezählt werden, wovon 265 aus dem unternehmerischen Bereich stammen und 74 aus dem akademisch wissenschaftlichen Umfeld. Diese Teilnahmen wurden letztlich von 242 Unternehmen und 57 Forschungseinrichtungen getragen, wodurch auch für die Qualifizierungsnetze Mehrfachteilnahmen einzelner Unternehmen und Forschungseinrichtungen erkennbar sind. 21 bzw. 10 Fördernehmer haben insgesamt zweimal an den QN-Projekten teilgenommen. 
Darüber hinaus sind einem Unternehmen drei Förderungen zuzurechnen bzw. zwei Forschungseinrichtungen drei Projektteilnahmen und einer vier Teilnahmen (siehe Grafik 11).

\section{Grafik 11 Teilnahme der Unternehmen und Forschungseinrichtungen an Qualifi- zierungsnetzen}

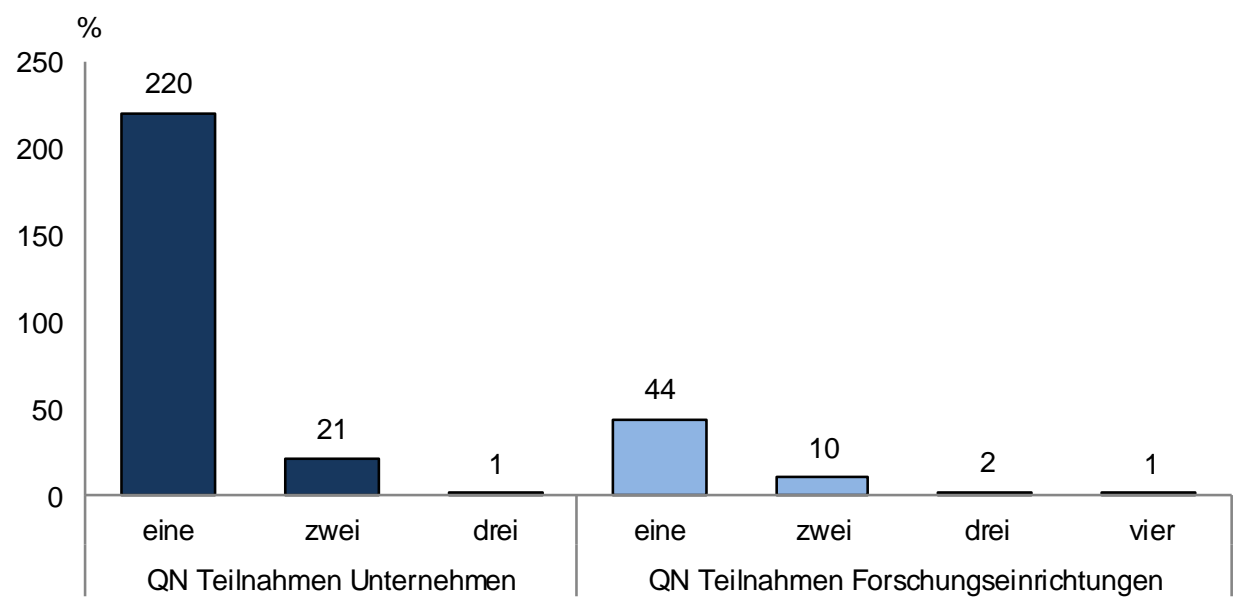

Quelle: eigene Darstellung, Daten FFG Monitoringdaten

Anmerkung: Hier wird nur die Förderschiene Qualifizierungsnetze betrachtet. Einige der Organisationen wurden auch im Rahmen der Qualifizierungsseminare oder der Innovationslehrveranstaltungen gefördert. Dies wird in Kap. 3.1 diskutiert.

In zwei Fällen lassen sich auch bei den Qualifizierungsnetzen Projektketten erkennen, die durch gleichbleibende Konsortien und aufbauende Projektinhalte gekennzeichnet sind. Ein weiteres Qualifizierungsnetz ergab ein Qualifizierungsseminar in einem anknüpfenden Themenbereich, abgewickelt durch ein überwiegend bestehendes Konsortium.

In den Qualifizierungsnetzen sind durchschnittlich 12,6 Unternehmen und 3,5 Forschungseinrichtungen zusammengefasst. Das kleinste Qualifizierungsnetz weist sechs Teilnehmer auf (fünf Unternehmen und eine Forschungseinrichtung), während das größte Netz aus 6 Forschungseinrichtungen und 32 Unternehmen besteht. Die Konsortialführung wird überwiegend von Forschungseinrichtungen getragen (rd. $86 \%$ ). Universitäten führen 7 der insgesamt 21 Konsortien als Projektleiter. In allen drei Projekten, die von Unternehmen geleitet werden, sind Kleinunternehmen als Konsortialführer eingesetzt.

Die thematischen Schwerpunktsetzungen der Qualifizierungsnetze sind die Bereiche Energie und Umwelt (7) sowie Produktion (8). Kumuliert umfassen diese mehr als zwei Drittel der Projekte. Weitere 4 Qualifizierungsnetze sind den IKT zuzurechnen und 2 den Life Sciences. 
Rund ein Drittel der Fördernehmer aus dem Unternehmensbereich ist in Niederösterreich beheimatet und weitere $29 \%$ in der Steiermark. Letztere zeichnet auch für den größten Anteil der Forschungseinrichtungen verantwortlich, ein Drittel der involvierten Forschungseinrichtungen stammt aus diesem Bundesland. Deutlich weniger Forschungseinrichtungen sind mit 18\% hingegen in Niederösterreich angesiedelt. Auf die übrigen sieben Bundesländer entfällt weniger als die Hälfte der verbliebenen Qualifizierungsnetzteilnahmen (siehe Grafik 12).

\section{Grafik 12 Regionale Verteilung der Fördernehmer der Qualifizierungsnetze}

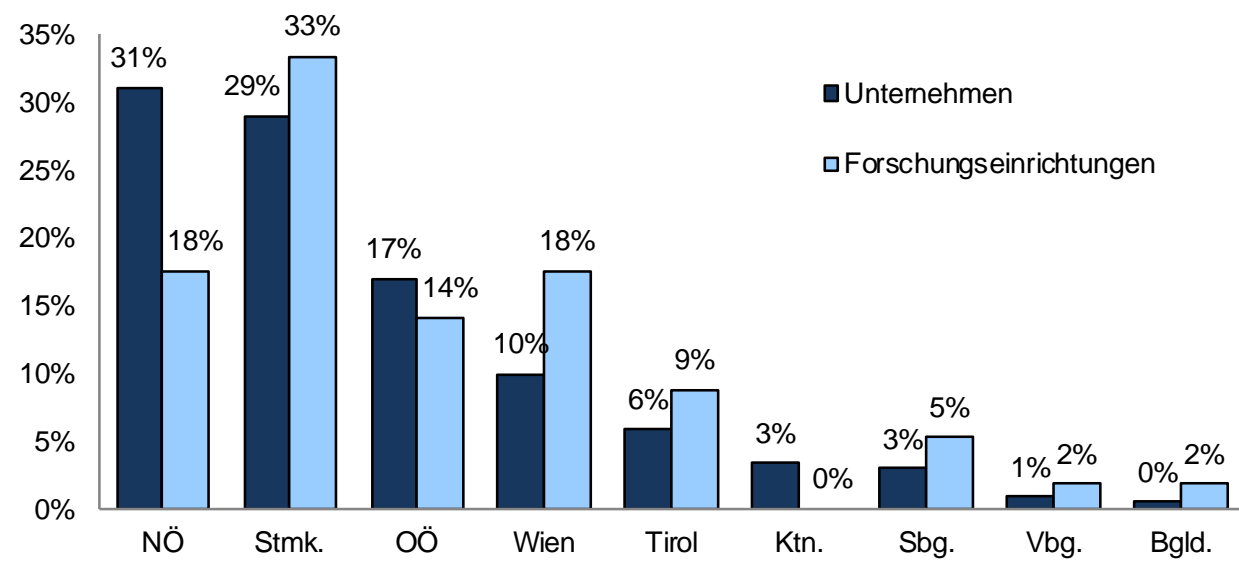

Quelle: eigene Darstellung, Daten: FFG Monitoringdaten

Der angestrebte Kompetenzaufbau in den Unternehmen drückt sich konsequenter weise im Verhältnis von Unternehmen und Forschungseinrichtungen aus. 81 \% der Fördernehmer gehören dem Unternehmenssektor an, die überwiegende Mehrheit davon sind Kleinunternehmen (140 bzw. 58 \%). Auf die Großunternehmen entfällt rd. ein weiteres Viertel (24\%) der geförderten Unternehmen, rd. 16\% sind Unternehmen mittlerer Größe.

95 der geförderten Unternehmen und Forschungseinrichtungen sind Neukunden der FFG, das entspricht rd. einem Drittel (32\%) der Fördernehmer der Qualifizierungsnetze. Der deutlich größere Anteil davon stammt aus dem Unternehmensbereich (87 Unternehmen bzw. $92 \%$ der Fördernehmer). Der Anteil der Kleinunternehmen beträgt dabei rd. $72 \%$, mehr als ein Viertel der Erstfördernehmer ist den mittleren oder Großunternehmen zuzuordnen. Wie auch bei den Qualifizierungsseminaren sind die FFG-neuen Forschungseinrichtungen (rd. $14 \%$ ) überwiegend außeruniversitäre Forschungseinrichtungen und Intermediäre. 


\section{Ziele und Motivation}

Die geförderten Qualifizierungsnetze verfolgten zwei wesentliche Zielbereiche. Zum einen waren dies die Steigerung der Innovationskompetenz und -fähigkeit der Unternehmen in (zukunfts-)relevanten Technologiefeldern sowie der Fachkompetenz und die Verbreitung des Know-Hows (aus verwandten Branchen) durch die Vermittlung von praktisch verwertbarem Wissen bzw. methodischen Zugängen. Zum anderen wollten die Qualifizierungsnetze zu einer nachhaltigen Vernetzung zwischen Wissenschaft und Wirtschaft, d.h. zwischen den teilnehmenden Betrieben und den wissenschaftlichen Partnern, bzw. zwischen Unternehmen verwandter Branchen / unterschiedlicher Gewerbe zur Bearbeitung von Schnittstellenproblematiken beitragen. Einzelne Qualifizierungsnetze beabsichtigten auch die (adaptierte) Qualifizierungsmaßnahme nachhaltig zu verankern. Dies korrespondiert mit den Ausschreibungsleitfäden, die als Ziele die Vernetzung von Wissenschaft und Forschung, das Ermöglichen eines Überblicks über relevante Technologiefelder, um innovative Technologiefelder besser antizipieren und davon profitieren zu können, und die Erhöhung der für Innovationen notwendigen Kompetenz und des Engagements der beteiligten Akteure als Zielbereiche beschreiben.

Die Motivation der befragten Unternehmen, an den Qualifizierungsnetzen teilzunehmen, war in erster Linie der Wissensaufbau, aber auch die Vertiefung und Verbreiterung des Wissens im Unternehmen. Mit dem Qualifizierungsnetz boten sich für die Unternehmen die Gelegenheit einer gezielten Ausbildung der MitarbeiterInnen und der Kontakt zu Wissenschaft und Forschung, zumal doch einige der Unternehmen zuvor noch nicht im Forschungsbereich tätig waren. Ein Unternehmer betonte zudem, dass solche qualitativ hochwertigen Weiterbildungen für Personen mit HTL-Matura oder Lehrabschluss sehr rar sind („Bisher mussten wir unsere Leute immer nach Deutschland schicken"). Die meisten interviewten Unternehmen betonten zudem, dass die Förderung der Personalkosten es innen erst ermöglicht hätte, die Schulungen überhaupt bzw. in dieser Intensität zu nutzen („Ohne Förderung wäre es finanziell nicht möglich gewesen“, „ohne Förderung nicht in diesem Umfang“, „Ich wäre auch ohne Förderung der Personalkosten hingegangen, meine MitarbeiterInnen hätte ich nicht hinschicken können").

\section{Ausgestaltung}

Zu Beginn der Projekte kam es meist zu inhaltlichen Abstimmungen der Curricula mit den Konsortialpartnern und einer genauen Festlegung der Ausbildungsinhalte oft im Rahmen von Steuerungsgruppen. Einige Unternehmen berichten, dass sich dies als durchaus herausfordernd dargestellt hat.

Die Schulungen waren meist in einer Art Modulstruktur aufgebaut, wobei jedes Modul, das oft mehrere Seminare/Workshops beinhaltete, einem Thema gewidmet war. So gliederte ein Netz seine Schulungen in vier Module mit Teilmodulen und zweitägigen Seminaren, wobei die ersten zwei Module der Vermittlung von Grund- 
lagen dienten, Modul 3 der Vertiefung und Modul 4 der Umsetzung des Erlernten (QN GrünAktivHaus). Im QN IQ-NET waren die 95 Schulungstage entlang dreier Technologiethemen und verschiedener Querschnittsthemen organisiert. Im QN F\&E_KKTER 2015 wurden 14 Einzelmodule mit 46 Schulungstagen drei großen Themenblöcken zugeordnet. Und das QN IQ-NET führte vier Qualifizierungsprogramme zu unterschiedlichen Themen durch.

Die Schulungen selbst beinhalteten meist einen Mix aus Vortrag, der die Vermittlung der Theorie zum Ziel hatte, sowie Diskussionen, Gruppenarbeiten und Übungen, bei denen das Erlernte Anwendung finden konnte. Die Praxisorientierung wurde in einigen Qualifizierungsnetzen dadurch unterstützt, dass in der Ausbildung konkrete Beispiele und Projekte aus den Unternehmen bearbeitet wurden (z.B. QN IQ-NET). In einem anderen Fall wurde das in Workshops erlangte Wissen in innerbetrieblichen Trainings umgesetzt und erprobt (QN Sichere F\&E) bzw. in einer betreuten Projektarbeit angewandt (QN KorrosionsExpert). Viele Qualifizierungsnetze richteten auch eine E-learning-Plattform ein, wo Unterrichtsmaterialien etc. verfügbar gemacht wurden. Im QN [QnHT] wurde auf ein Blended Learning Konzept gesetzt: Präsenzblöcke wechselten mit Onlinephasen ab. Einige Qualifizierungsnetze planten auch zwei Durchführungsphasen und adaptierten die zweite Phase auf Basis von in der ersten Phase gewonnenen Erfahrungen (QN RFiD).

Vortragende waren meist Mitarbeiterlnnen der wissenschaftlichen Partner oder externe Vortragende, teilweise erfolgte der Wissenstransfer in den Schulungen auch multilateral, wenn Konsortialmitglieder als Lehrende und Lernende fungierten (QN GrünAktivHaus).

Insgesamt wurden im Zuge der ersten Ausschreibung 618 Personen ausgebildet, wovon 101 (rd. $16 \%$ ) weiblich waren. Der Anteil der Teilnehmerinnen in dieser Ausschreibung pendelt zwischen $50 \%$ und $0 \%$ (ein Projekt), wobei drei Projekte einen hohen Frauenanteil von über $25 \%$ aufweisen (siehe Grafik 13). Im Rahmen der zweiten Ausschreibung wird die Qualifikation von 596 Personen bei einem etwa gleichbleibenden Frauenanteil (rd. $15 \%$ ) angestrebt. In allen Qualifizierungsnetzen ist die Teilnahme von Frauen geplant (siehe Grafik 14).

Die Teilnehmerlnnen wurden meist aufgrund von Vorgesprächen auf Basis ihrer Interessen und Entwicklungsperspektiven und auch in Absprache mit AbteilungsleiterInnen von der Geschäftsführung ausgewählt. Die Teilnahme von Frauen hängt sehr stark von der Branche, in der die Unternehmen tätig sind, ab und variiert auch in Abhängigkeit von den Schulungsinhalten. 
Grafik 13 Teilnehmerlnnenanzahl und Frauenanteil der Qualifizierungsnetze der ersten Ausschreibung

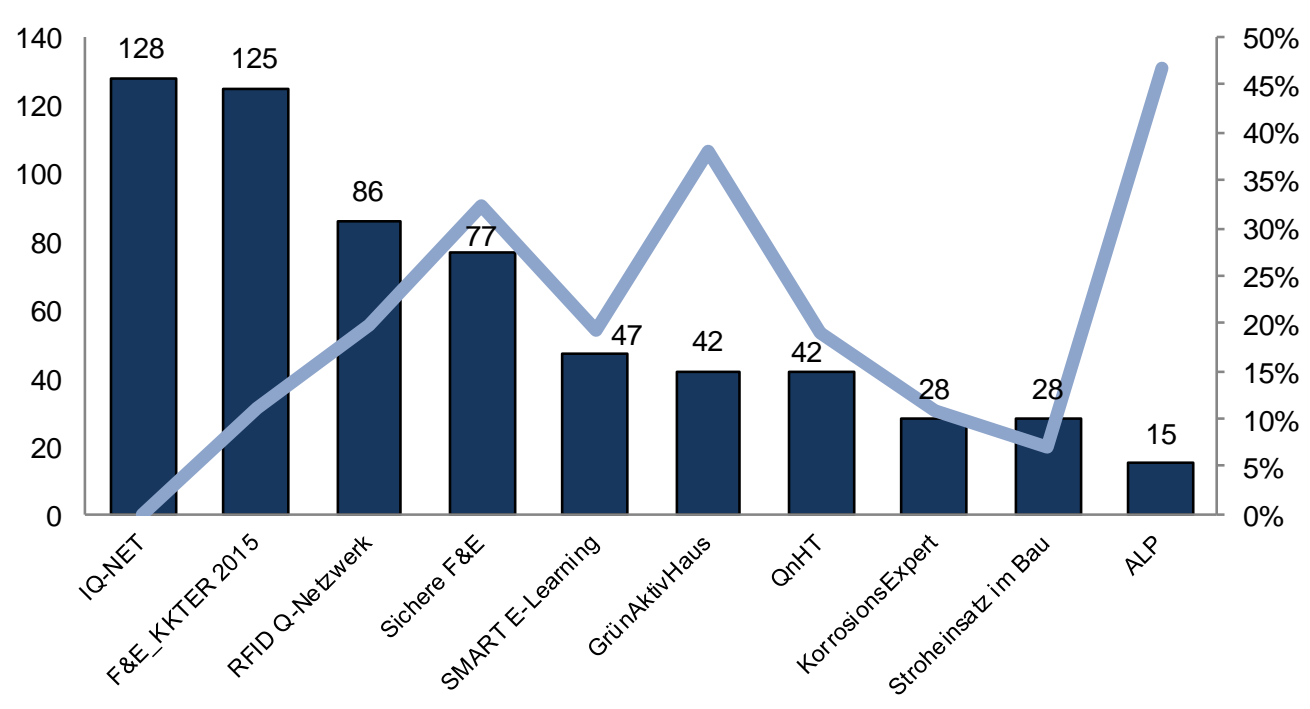

Quelle: eigene Darstellung, Daten: interne Auswertung FFG

Grafik 14 Vorläufige Teilnehmerlnnenanzahl und Frauenanteil der Qualifizierungsnetze der zweiten Ausschreibung

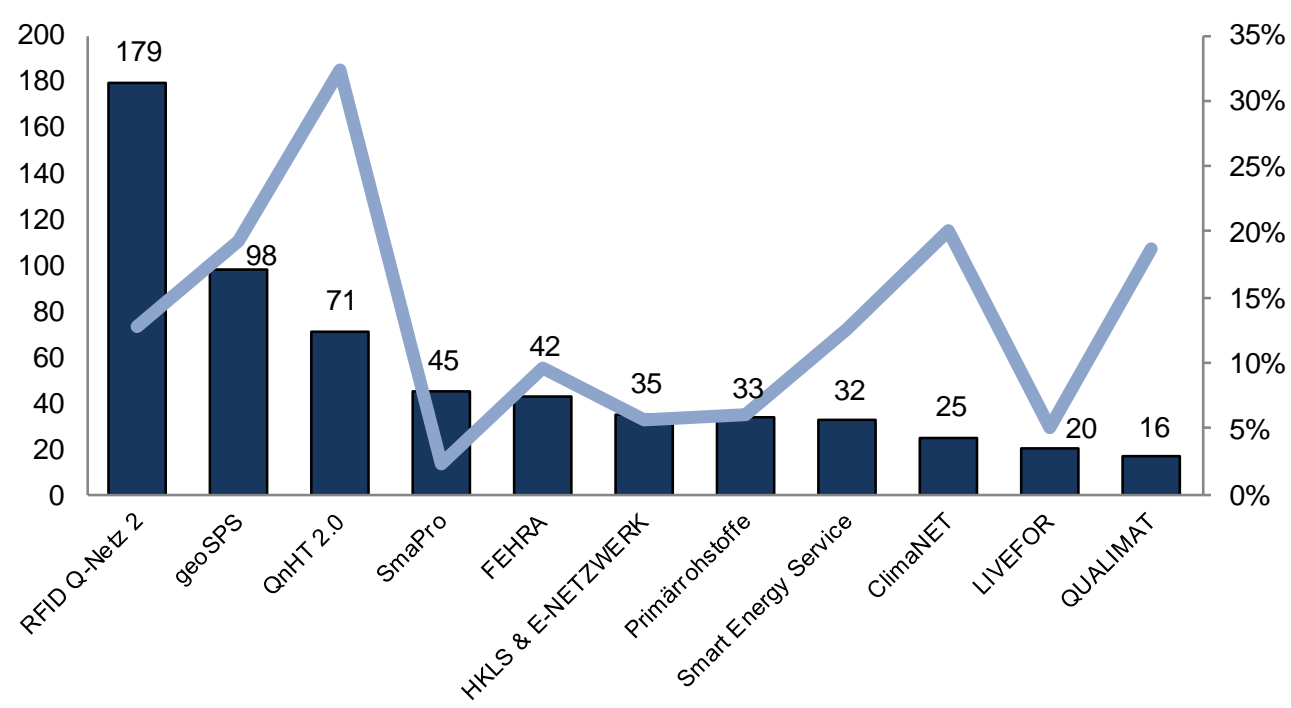

Quelle: eigene Darstellung, Daten: interne Auswertung FFG 
Feedback der Teilnehmerlnnen wurde meist am Ende jeder Schulungseinheit oder am Ende jedes Moduls mittels Feedbackfragebogen und auch in persönlichen Gesprächen eingeholt. Dabei wurden die Kurse meist mit sehr gut und gut bewertet. Zudem fiel die Gesamtbeurteilung insgesamt sehr positiv aus, dies geht auch aus den Besuchen vor Ort durch die FFG hervor. Auch die interviewten Unternehmen unterstrichen die hohe Qualität der Ausbildung und lobten die profunde Auseinandersetzung mit der Thematik („Das ging wesentlich tiefer als die Kurse, die wir bisher gemacht haben") sowie den gegebenen Praxisbezug („Praxisbezug war immer da, greifbar").

Dennoch wurden einige Aspekte als kritisch gesehen und stellten Herausforderungen bei der Durchführung der Schulungen dar:

- Heterogenität der TeilnehmerInnen: Einige Gruppen waren sehr heterogen in Bezug auf Vorwissen, Ausbildung oder Berufserfahrung. Dabei konnte bei branchenübergreifenden Qualifizierungsnetzen damit gerechnet werden, aber auch branchenhomogenere Netze waren damit konfrontiert, dass z.B. „alte Hasen“ neben unerfahrenen Mitarbeiterlnnen an der gleichen Schulung teilnahmen. So wurden z.B. Orientierungsworkshops den eigentlichen Schulungseinheiten vorgeschaltet oder ein nicht geplantes Seminar durchgeführt, um eine gemeinsame Wissensbasis zu schaffen.

- E-learning: E-learning wurde von den Teilnehmerlnnen teilweise kritisch beurteilt und nicht so angenommen wie von den wissenschaftlichen Partnern geplant. Dies zeigt, dass methodische Vorgehensweisen, wie sie im tertiären Bereich gang und gäbe sind, nicht unreflektiert auf betriebliche Weiterbildungen übertragen werden können.

- Vereinbarkeit mit dem Berufsalltag: Die (mehrtägigen) Schulungen, die sich über einen längeren Zeitraum erstrecken, bedeuten die Abwesenheit von MitarbeiterInnen im Betrieb. Dies stellt v.a. für kleine Unternehmen eine große Herausforderung dar, da eine bestimmte Expertise teilweise nur ein/e Mitarbeiterln mitbringt und diese/r dann oft kurzfristig im Betrieb nicht abkömmlich ist. Damit kam es im Rahmen der Qualifizierungsnetze immer wieder zu kurzfristigen Ausfällen der TeilnehmerInnen oder zur Verschiebung von Schulungsterminen. Um dem entgegenzuwirken, wurde auf eine sehr langfristige Planung, Erinnerungen per Mail oder Telefon etc. gesetzt, ebenso wie auf die Etablierung von Wartelisten. 
- Vorbehalte gegenüber anderen Unternehmen: Bei den Besuchen vor Ort durch die FFG wurde deutlich, dass die Teilnehmerlnnen teilweise wenig Einblick in andere Unternehmen bekommen. Hier dürfte es Vorbehalte gegeben haben, zu viel vom eigenen Betrieb preiszugeben, was u.a. durch eine direkte Konkurrenzsituation erklärt werden kann. Auch dürfte der Austausch mit anderen Unternehmen auf Ebene der Schulungsteilnehmerlnnen in einigen Netzen nicht in gewünschtem Ausmaß erfolgt sein. Auf Unternehmensführungsebene dürfte dieser Austausch dagegen gelungen sein, wie die interviewten Geschäftsführer berichten. Dies mag auch daran liegen, dass auf der Ebene der MitarbeiterInnen Unklarheit darüber herrscht, welche Informationen aus dem eigenen Unternehmen preisgegeben werden dürfen.

\section{Kooperationen}

Die Kooperation zwischen den Konsortialpartnern ist in den Qualifizierungsnetzen gut verlaufen. Zentrales Element war in den meisten Netzen eine Steuerungsgruppe bestehend aus dem Kernteam (Konsortialführung und wissenschaftliche Partner) und VertreterInnen der beteiligten Unternehmen. Diese trafen sich meist regelmäßig, zu Beginn gab es ein Kick-off Meeting. Die Abstimmungstreffen dienten der Detailplanung der Schulungen und in weiterer Folge der Diskussion über weiterführende Projekte.

In manchen Fällen trafen sich die Projektpartner v.a. im Rahmen der Schulungen. Abstimmungen erfolgten dann mehr bilateral (z.B. zwischen wissenschaftlichen Partnern oder mit ReferentInnen). Teilweise wurden auch Veranstaltungen organisiert, in denen sich Forschungseinrichtungen vorgestellt haben oder ExpertInnen referierten. In einem Fall verlief die Kommunikation auch online über Intranet.

In allen Projekten kannten sich die Projektpartner zumindest teilweise, v.a. hatte die Konsortialführung schon meist im Vorfeld Kontakt mit den (anderen) wissenschaftlichen Partnern und einigen beteiligten Unternehmen (ein interviewter Unternehmer ist z.B. als Lektor an einer Universität tätig). Auch kannten sich schon einige Unternehmen bevor das jeweilige Qualifizierungsnetzwerk ins Leben gerufen wurde.

Einige Qualifizierungsnetze mussten den Ausstieg von einzelnen Partnern verkraften bzw. erwiesen sich einige Partner als inaktiv in dem Sinne, als diese keine TeilnehmerInnen zu den Schulungen entsandten. Auch kam es in einigen Fällen zu Änderungen der Ansprechpartnerln, was die Organisation erschwerte.

Die interviewten Unternehmen konnten durch die Teilnahme am Qualifizierungsnetz ihre Verbindungen zu den wissenschaftlichen Partnern intensivieren. Ein Unternehmer berichtet z.B., dass er in Zukunft bei bestimmten Berechnungen die 
Universität heranziehen werde. Mit den anderen Unternehmen gibt es teilweise Gespräche über zukünftige Kooperationen bzw. sind diese bereits in der Planungsphase, wenngleich nicht immer direkte Berührungspunkte gegeben sind. Es bestehe loser Kontakt („es verflüchtigt sich - leider"). In jedem Fall trugen aber einige Qualifizierungsnetze dazu bei, unter den Unternehmen eine Vertrauensbasis zu schaffen und Ängste auszuräumen (,Ich traue mich jetzt, diese Firmen auch weiterzuempfehlen").

\section{Wirkungen}

Folgende Wirkungen auf die beteiligten Akteure konnten identifiziert werden:

- Kompetenzerweiterung und Stärkung der Innovationskompetenz: Die Unternehmen berichten von einer Steigerung ihrer Innovationskompetenz und der höheren Qualifizierung ihrer MitarbeiterInnen. Diese können die Unternehmen im Kontakt mit den KundInnen durch kompetenteres Auftreten unmittelbar nützen ("Sie können jetzt auf Augenhöhe kommunizieren"). Dies betrifft z.B. auch Medienauftritte (QN Sichere F\&E). In einigen Fällen wurde der Kompetenzaufbau auch durch interne Schulungen auf Basis des Erlernten verstärkt bzw. ist die Gründung einer Weiterbildungsakademie in Planung. Die Teilnahme an den Schulungen habe aber auch zu einem Motivationsschub geführt („Die sind stolz, dass sie das geschafft haben.“), der wiederum der Innovationskompetenz zuträglich ist.

- Weiterentwicklung der Qualifizierungsangebote: Es konnten einige nachhaltige Qualifizierungsangebote konzipiert werden, die teilweise bereits angeboten werden. Im Rahmen von QN IQ-NET wurde ein modular aufgebautes, tertiäres Ausbildungsmodell erarbeitet. Die 8 Module können zu 5 verschiedenen akademischen Lehrgängen und 2 Masterlehrgängen kombiniert werden. Die berufsbegleitenden Lehrgänge werden bei zwei Netzwerkpartnern akkreditiert und starteten im September 2014 mit 45 Studierenden. Ein beteiligter Cluster bietet die erarbeiteten Module in Form von Tagesschulungen und Inhouse-Seminaren an (QN F\&E_KKTER 2015). Aus dem QN ALP ging ein Workshopangebot hervor (Tagesschulung, 3-Tagesschulung, Vortrag, Workshop). Die Projektpartner sollen dieses Angebot über ihre Netzwerke promoten, um den Workshopplan 2015 zu realisieren. In einem anderen Fall befindet sich ein berufsbegleitender Universitätslehrgang in Entwicklung (QN KorrosionsExpert). Der Verein, der aus GrünAktivHaus entsteht (siehe unten), plant eine Ausbildungsschiene für Fassadenbegrünung, die im Forschungs- und Kompetenzzentrum für Bauen und Energie Großschönau angeboten werden soll. Einige Fachhochschulen nützen die Ergebnisse zur Verbesserung der wissenschaftlichen Lehre, da diese über die Qualifizierungsnetze einen Einblick in die Belange der Wirtschaft erhalten konnten. 
- Networking: Aus einigen Qualifizierungsnetzen gingen nachhaltige Netzwerke hervor oder wurden in bestehende integriert bzw. wurden weitere Netzwerktreffen organisiert (z.B. QN IQ-NET; regelmäßige Arbeitsgruppentreffen, Forschungs- und Technologieforum 2014; QN Sichere F\&E: Jährliche Netzwerktreffen mit größerem Teilnehmerlnnenkreis). Die meisten Partner von GrünAktivHaus gründen einen Verein, damit das Netzwerk weiterlebt. Auch sind alle Partner dem Verband für Bauwerksbegrünung beigetreten und haben damit auch eine europaweite Anbindung. Mehrere Unternehmen des Qualifizierungsnetzes treten zudem als Bietergemeinschaft für ein Bauvorhaben auf bzw. zwei Partner wollen gemeinsam bei einem Wettbewerb antreten. Auch wurde ein Spin-off Unternehmen gegründet, das Forschung, Beratung und Training anbietet.

- Produktentwicklung: Die Teilnahme an den Qualifizierungsnetzen brachte den Unternehmen neue Impulse und Ideen, die für die Produktentwicklung essentiell sind. Einige Unternehmen haben Innovationsprozesse neu aufgestellt und nützen das Wissen zur Erlangung von Zertifizierungen (QN Sichere F\&E). Andere haben Konstruktionsänderungen vorgenommen oder Prozessketten optimiert bzw. bestehende Produkte verbessert (QN KorrosionsExpert). Die Entwicklung eines Leuchtturmprojekts, das auch jetzt zugänglich ist und den Stand der Technik anschaulich macht (Umgestaltung eines Gebäudes: Dachbegrünung mit Fotovoltaik, vertikale erdungebundene Begrünung etc.) stand u.a. im Fokus von GrünAktivHaus.

- Folgeprojekte: Konkrete F\&E-Folgeprojekte gibt es noch wenige (z.B. IQNET: Analytikdatenbank). Allerdings kam es z.B. in einem Fall zu einer Projekteinreichung mit mehreren Partnern eines Qualifizierungsnetzes, das Projekt wurde jedoch nicht genehmigt.

\section{Beurteilung}

Ebenso wie die Qualifizierungsseminare finden die Qualifizierungsnetze bei den beteiligten Unternehmen großen Anklang und werden als ausgezeichnetes Instrument der Weiterbildung der MitarbeiterInnen gesehen, die in der Folge die Innovationskompetenz der Unternehmen stärkt. Dies wird auch darin deutlich, dass ein interviewtes Unternehmen mit einer Fachhochschule konkrete Gespräche über ein Qualifizierungsnetz mit neuem Schwerpunkt führt und andere KMU für die Teilnahme sucht. Ein anderes Unternehmen berichtet von einem abgelehnten Antrag in der 2. Ausschreibungsrunde. Rd. jedes zehnte Unternehmen nahm bzw. nimmt an mehr als einem Qualifizierungsnetz teil.

Stärken bzw. Erfolgsfaktoren der Qualifizierungsnetze sind: 
- Die Schulungsinhalte werden an die Bedürfnisse der teilnehmenden Unternehmen angepasst. Die Erprobung des Gelernten an konkreten Beispielen im eigenen Unternehmen stößt dabei auf besonderen Anklang.

- Die Kompetenz der Vortragenden und wissenschaftlichen Partner ist gegeben und trägt in erheblichem Maße zum Erfolg bei.

- Der Vernetzungsaspekt wird einhellig begrüßt. Die Einbringung von verschiedenen Perspektiven und die Bearbeitung von Schnittstellen werden als sehr positiv empfunden („Die Vielfalt der Teilnehmer war sehr bereichernd.")

- Die Verzahnung von Wissenschaft und Praxis bzw. die Zusammenführung des Stands der Wissenschaft mit dem Stand der Technik und dem KnowHow der Ausführenden ist eine Stärke des Instruments („So kann gegenseitiges Verständnis geschaffen werden und durch die Rückkoppelung neue Erkenntnisse gewonnen werden"). Auch die Intensivierung des Kontakts zu den wissenschaftlichen Partnern wird als positive Folge des Projekts identifiziert.

Daneben gibt es aber auch einige Herausforderungen:

- Die verschiedenen Unternehmen aus verschiedenen Bereichen müssen ein gemeinsames Ziel formulieren („Die muss man erst einmal alle unter einen Hut bringen").

- Die Qualifizierungsnetze werden als forderndes Instrument bezeichnet. Der Zeitaufwand für die TeilnehmerInnen ist hoch (auch am Wochenende) und nicht alle MitarbeiterInnen waren motiviert, sich über einen längeren Zeitraum zu einer solchen Weiterbildungsmaßnahme zu verpflichten.

- Die Nennung der Teilnehmerlnnen am Beginn des Projekts stellt v.a. für kleinere Betriebe ein Problem dar, weil kurzfristige Kundenanfragen schnell zu Kapazitätsengpässen führen können und eine langfristige Planung teilweise schwer möglich ist.

Einige Qualifizierungsnetze scheinen insbesondere in Hinblick auf die Etablierung von Qualifizierungsmaßnahmen erfolgreich. Damit steht auch anderen Unternehmen ein bereits erprobtes Weiterbildungsprogramm zur Verfügung und es kommt zu einer nachhaltigen Verankerung von in den Qualifizierungsnetzen erarbeiteten Inhalten in Qualifizierungsangeboten. Damit wird ein Beitrag zur Steigerung der Innovationskompetenz über die beteiligten Unternehmen hinaus geleistet. 


\subsubsection{Innovationslehrveranstaltungen}

Die Innovationslehrveranstaltungen stellen quasi die „Königsklasse“ im Rahmen des Förderschwerpunkts dar. Im Rahmen der Innovationslehrveranstaltungen werden längerfristige Qualifizierungsnetzwerke in neuralgischen und derzeit unterbesetzten Themenfeldern gefördert. Zielgruppe sind technologisch kompetente Unternehmen und forschungsorientierte Unternehmen mit eigener F\&E-Abteilung. Ziel der Maßnahme ist die Unterstützung des systematischen Aufbaus und der Höherqualifizierung des vorhandenen Forschungs- und Innovationspersonals, die stärkere Verankerung unternehmensrelevanter Lehr- und Forschungsschwerpunkte in tertiären Bildungseinrichtungen sowie die nachhaltige Etablierung von bisher nicht adressierten, wirtschaftsnahen Themen im Qualifizierungsangebot.

Tabelle 3 Eckpunkte der Innovationslehrveranstaltungen

\begin{tabular}{|l|l|}
\hline \multicolumn{1}{|c|}{$\begin{array}{c}\text { Innovationslehr- } \\
\text { veranstaltungen }\end{array}$} & \multicolumn{1}{|c|}{ Kurzbeschreibung } \\
\hline \multirow{2}{*}{ Ziele } & $\begin{array}{l}\text { Unterstützung von Unternehmen im systematischen Aufbau und } \\
\text { der Höherqualifizierung des vorhandenen Forschungs- und Inno- } \\
\text { vationspersonals } \\
\text { Beitrag zu einer stärkeren Verankerung unternehmensrelevanter } \\
\text { Lehr- und Forschungsschwerpunkte an österreichischen Universi- } \\
\text { täten und Fachhochschulen sowie zur Erhöhung der vielfach } \\
\text { bemängelten sektoralen Mobilizät } \\
\text { Nachhaltige Etablierung von bisher nicht adressierten, wirt- } \\
\text { schaftsnahen Themen im Qualifizierungsangebot }\end{array}$ \\
\hline Zielgruppen & $\begin{array}{l}\text { Technologisch kompetente Unternehmen } \\
\text { Forschungsorientierte Unternehmen (Unternehmen mit eigener } \\
\text { F\&E-Abteilung, dass in der Lage ist, sich längerfristig zu engagie- } \\
\text { ren) }\end{array}$ \\
\hline Konsortium & $\begin{array}{l}\text { Gefördert werden längerfristige, zeitlich begrenzte Qualifizie- } \\
\text { rungsnetze in neuralgischen und derzeit unterbesetzten Themen- } \\
\text { feldern. Das Angebot muss in Kooperation zwischen Universitä- } \\
\text { ten/Fachhochschulen und Unternehmen aufgesetzt werden. Die } \\
\text { Themensetzung folgt dem Qualifizierungsbedarf des Unterneh- } \\
\text { mensnetzwerks und fokussiert auf Themenfelder, in denen sys- } \\
\text { tematische qualitative und quantitative Defizite im Qualifizie- } \\
\text { rungsangebot vorliegen. }\end{array}$ \\
\hline Laufzeit & $\begin{array}{l}\text { 1 Ausschreibung Oktober 2012 - März 2013 } \\
\text { Förderhöhe } \\
\text { min. 3 Unternehmenspartner (davon min. 3 KMU) und 1 wissen- } \\
\text { schaftlicher Partner }\end{array}$ \\
\hline Min. 24 bis max. 48 Monate \\
\hline Max. € 1.000.000,-
\end{tabular}


Im Rahmen der Innovationslehrveranstaltungen werden bis September 2017 drei Projekte mit 42 teilnehmenden Organisationen bzw. 43 Beteiligungen (eine Forschungseinrichtung ist in zwei Lehrveranstaltungen vertreten) gefördert. Zur Förderung eingereicht wurden bis dato im Rahmen der einzigen Ausschreibung acht Lehrveranstaltungen, das bedeutet, dass der Anteil der geförderten Lehrveranstaltungen bei etwa einem Drittel liegt ( $r d .38 \%)$. Eine projektübergreifende Fortführung der Zusammenarbeit von Lehrveranstaltungsteilnehmern drückt sich in einem anknüpfenden Qualifizierungsnetz aus.

36 der 42 involvierten Fördernehmer (rd. $86 \%$ ) sind Unternehmen, die anderen 6 Forschungseinrichtungen. Davon sind wiederum mehr als die Hälfte (57\%) Kleinunternehmen. Die Teilnahmen mittlerer Unternehmen (7) und von Großunternehmen (5) halten sich in etwa die Waage (siehe Grafik 15).

In den Innovationslehrveranstaltungen sind durchschnittlich 12 Unternehmen und 2,3 Forschungseinheiten zusammengefasst. Die umfangreichste Lehrveranstaltung weist 19 Beteiligungen auf, die sich aus 17 Unternehmen und 2 Forschungseinrichtungen zusammensetzt. Die kleinste der drei Innovationslehrveranstaltungen besteht aus 10 Teilnehmern, von denen 7 Unternehmen sind. In allen Projekten sind wissenschaftliche Partner die Konsortialführer, in zwei sind es Fachhochschulen bzw. in einem Projekt eine Universität.

Zwei Drittel der Innovationslehrveranstaltungsteilnehmer sind in Oberösterreich bzw. Wien ansässig (jeweils 14 Unternehmen und FE). Darüber hinaus beteiligen sich Fördernehmer aus dem Burgenland (4), Steiermark (3), Niederösterreich (3), Salzburg (1) und Tirol (1) (siehe Grafik 15). Die regionale Verteilung der Teilnehmer ist vorwiegend der Herkunft der lehrenden Forschungseinrichtungen (Burgenland, Oberösterreich, Wien) geschuldet.

\section{Grafik 15 Größenklasse und Herkunft der Fördernehmer der Inno-LV}
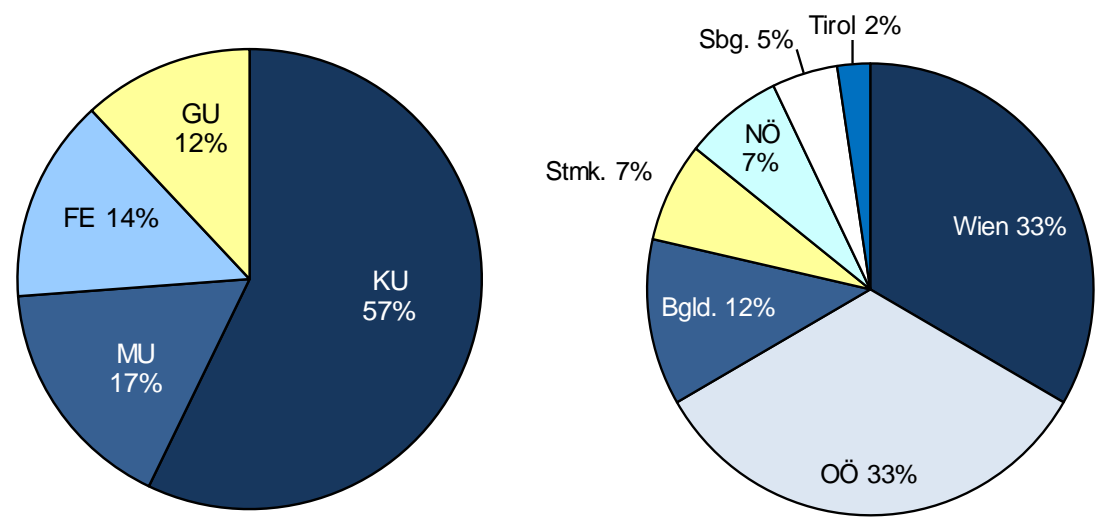

Quelle: eigene Darstellung, Daten: FFG Monitoringdaten 
Thematisch können die drei Lehrveranstaltungen den Bereichen Energie und Umwelt, IKT und Sonstige zugeordnet werden.

Von den teilnehmenden Organisationen haben 6 (14\%) die Innovationslehrveranstaltung für den Einstieg in das Förderportfolio der FFG gewählt. Wenig überraschend sind diese ausschließlich Kleinunternehmen und daher dem Unternehmensbereich zuzurechnen. Dementsprechend sind rd. $17 \%$ der beteiligten Unternehmen Erstfördernehmer.

Die 30 bestehenden FFG Kunden aus dem Unternehmensbereich waren vor der Projektdurchführung vor allem im Bereich der Basisprogramme aktiv. Im Rahmen dieser Förderlinie wurden insgesamt 89 Beteiligungen abgewickelt. Weitere Förderungen wurden im Rahmen von Talente, COMET, generation innovation Praktika und dem Innovationsscheck abgewickelt. Die befragten Unternehmen berichten teilweise darüber hinaus auch von Förderungen anderer Agenturen.

\section{Ziele und Motivation}

Die drei Lehrveranstaltungen zielen auf Kompetenzerweiterungen von Unternehmen in Schlüsseltechnologien vor dem Hintergrund neuer Rahmenbedingungen (Energiespeicher - eNNOVATION) bzw. in Themenfeldern, die für die Unternehmen an Relevanz gewinnen (Security, Privacy, Malware - MAPO) oder die Bildung eines branchenübergreifenden Systemdenkens zur Bewältigung zukünftiger Herausforderungen (Industrie 4.0 - McTronic). Damit widmen sich alle drei Projekte aktuellen Herausforderungen der involvierten Branchen, die zwar von großer Aktualität sind, aber im derzeitigen tertiären Ausbildungsangebot nicht (ausreichend) repräsentiert sind bzw. auch Standardschulungen keine Option darstellen, da es sich um Spezialthemen („In dieser Spezialisierung gibt es keine allgemeinen Schulungen.") oder extrem dynamische Bereiche handelt. Die Themen sind für die teilnehmenden Unternehmen meist nicht neu bzw. handelt es sich teilweise um ihr Kerngeschäft, aber es besteht großer Bedarf an einer Vertiefung (,wird immer breiter, ändert sich ständig") bzw. einem ständigen Update in diesem Bereich („Wir brauchen hier permanent Input.").

In allen drei Projekten sollen zudem Unternehmen verschiedener Branchen bzw. Geschäftsfelder ein gemeinsames Verständnis für Problembereiche und ganzheitliche Lösungen erarbeiten.

Die Motivation zur Teilnahme war bei den wissenschaftlichen Partnern inhaltlich getrieben („,brauchen neuen Lösungen“, „Dinge anschauen, für die sonst keine Zeit ist"). Die Unternehmen sehen in der Teilnahme eine Investition in die Zukunft („Firmen müssen in Vorleistung gehen.“; „Wie rüsten wir uns für die Digitalisierung?"), die Möglichkeit zum Wissensaufbau (aber auch zur Verbreiterung bzw. Auffrischung des Wissens) auch in neuen Bereichen („,die Mitarbeiter auf den neuesten Stand der Technik bringen"), aber auch die Möglichkeit zur Vernetzung in 
der jeweiligen Szene und zum Erfahrungsaustausch („Spezialisten müssen sich hier austauschen."). Bei zwei der Innovationslehrveranstaltungen waren eher die wissenschaftlichen Partner treibende Kraft hinter dem Projekt, die die Unternehmen gezielt angesprochen haben, eine Innovationslehrveranstaltung wurde von einem Unternehmenspartner initiiert, der den wissenschaftlichen Partner ins Boot geholt und dann zusammen mit diesem die anderen Unternehmen rekrutiert hat.

Auch bei den Innovationslehrveranstaltungen ist nicht die Förderung der Personalkosten ausschlaggebend für die Teilnahme der Unternehmen (einige der befragten Unternehmen hätten auch ohne diese an der Innovationslehrveranstaltung teilgenommen), sondern in erster Linie das attraktive Schulungsangebot („Hier bekommen wir Zugang zu Leuten und zu Wissen, wo wir sonst nicht so leicht drankommen."). Bei MAPO, wo die Unternehmensvertreter je nach Thema in die Rolle des Teilnehmers oder Vortragenden schlüpfen, wird betont, dass die Vortragstätigkeit nur mit einer Förderung der Personalkosten möglich ist, die reine Teilnahme sei aber auch ohne diese denkbar.

\section{Ausgestaltung}

Die konkrete Ausgestaltung der Schulungen wurde zumeist im Vorfeld mit den Unternehmen diskutiert. Alle Innovationslehrveranstaltungen setzen bei den Schulungen auf eine Mischung aus Vorträgen und praktischen Übungen. Die TeilnehmerInnen sollen „hands-on“ mit Versuchsaufbauten arbeiten können. Teilweise arbeiten die Teilnehmer der verschiedenen Unternehmen an praktischen Aufgaben in virtueller Umgebung, wo unterschiedliche Rollen eingenommen werden („Angreifer" und "Verteidiger"). Die Unternehmen schätzen die praktischen Übungen, den Wechsel von Vortrag und interaktiven Übungen sowie die Diskussionsmöglichkeiten im Rahmen der Schulungen, die als sehr befruchtend wahrgenommen werden. Schulungen mit Workshopcharakter erhalten denn auch das beste Feedback. Im Projekt MAPO erwies sich die „Hackweek“ als Highlight.

Das Bestreben, die Schulungen sehr praxisorientiert und wirtschaftsnah anzubieten, spiegelt sich auch bei der Auswahl der Vortragenden wider. Bei MAPO werden die Vortragenden aus den Unternehmen des Konsortiums rekrutiert, d.h. je nach Themenstellung stellt ein spezialisiertes Unternehmen den Vortragenden. Damit kommt es zu einem optimalen Wissensaustausch mit dem Ziel, dass am Ende des Projekts alle Unternehmen den gleichen Wissensstand haben. So haben alle Konsortialpartner die Rolle als Lehrende und Teilnehmende inne. Eine Innovationslehrveranstaltung setzt v.a. auf ExpertInnen aus der Wirtschaft (eNNOVATION). Bei McTronic schlagen auch Unternehmen externe Vortragende vor.

Zwei Innovationslehrveranstaltungen sehen das Programm grundsätzlich für alle teilnehmende Unternehmen vor, eNNOVATION bietet Grundmodule, die alle Unternehmen besuchen sollen und Spezialmodule, aus denen dann eine Auswahl getroffen wird. 
Die Teilnehmerlnnen werden nach Interesse ausgewählt bzw. in Abhängigkeit der Themenfelder, die die einzelnen Personen im Unternehmen bearbeiten (sollen). Daher besuchen meist auch unterschiedliche Personen die einzelnen Schulungsmaßnahmen in Abhängigkeit der jeweiligen Schulungsinhalte. Manche Unternehmen nominieren einen Kern an Personen, die an fast allen Schulungen teilnehmen und dann fallweise von anderen Personen begleitet werden. Ein befragtes Unternehmen wird nur vom Geschäftsführer vertreten. In der Folge erfolgt aber zunächst in den befragten Unternehmen ein breiter, wenn auch meist informeller Wissenstransfer.

Im Rahmen der drei Projekte sollen insgesamt 169 Personen qualifiziert werden, davon sind 16 (rd. $9 \%$ ) weiblich. Wie sich aus untenstehender Abbildung (siehe Grafik 16) erkennen lässt, liegt der Frauenanteil unter den TeilnehmerInnen in allen Projekten unter $20 \%$. Die Lehrveranstaltung, die dem Bereich der IKT zuordenbar ist, wird gänzlich ohne weibliche Beteiligung durchgeführt, während die Lehrveranstaltung aus dem Bereich Energie und Umwelt zumindest einen Frauenanteil von $16 \%$ anstrebt.

\section{Grafik 16 Geplante TeilnehmerInnenzahl und Frauenanteil der Innovationslehrver- anstaltungen}

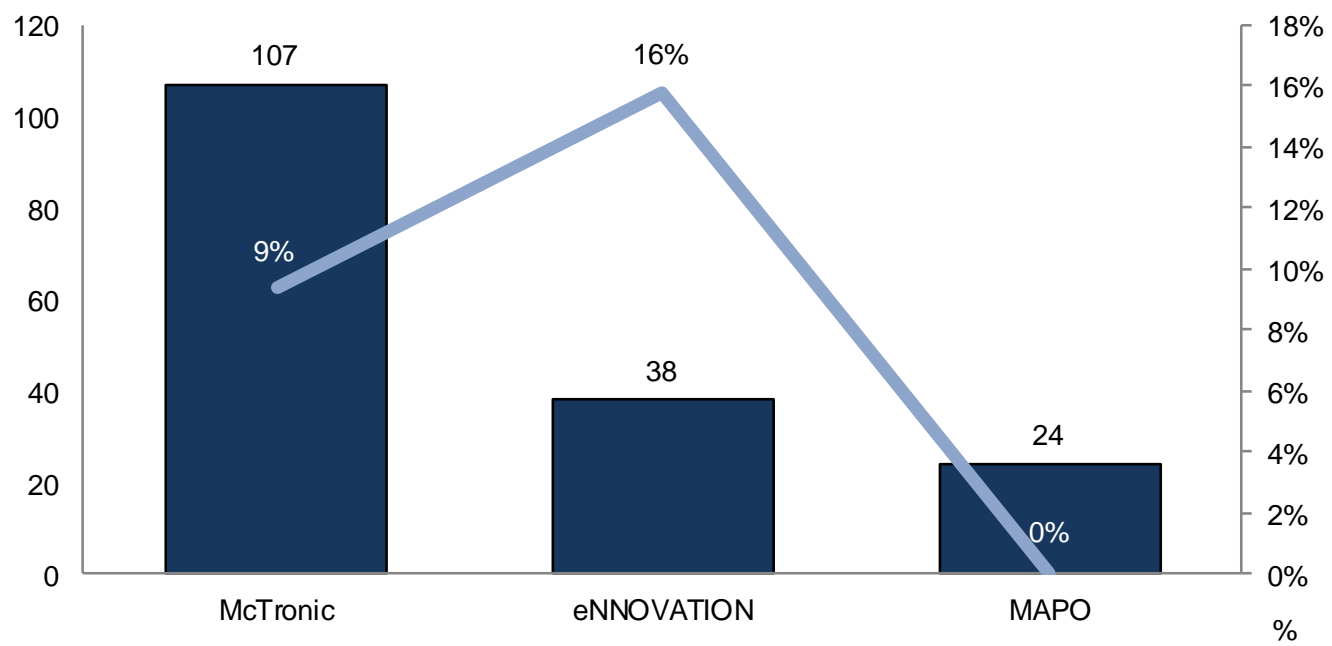

Quelle: eigene Darstellung, Daten: interne Auswertung FFG

Der geringe Frauenanteil spiegelt auch hier die niedrige Frauenquote in den relevanten Branchen wider. Zudem spielt das spezifische Thema der Lehrveranstaltungen eine wesentliche Rolle („Unter den InformatikstudentInnen sind schon weniger als $20 \%$ Frauen, Security ist eine reine Männerdomaine. "). 
Die Evaluierung der Schulungen erfolgt unterschiedlich. Ein Projekt setzt auf das Einholen schriftlichen, standardisierten Feedbacks nach jeder Einheit, während es in einem anderen Fall zu informellen Gesprächen zwischen wissenschaftlicher Leitung und Teilnehmerlnnen bzw. GeschäftsführerInnen der teilnehmenden Unternehmen kommt. Die im Rahmen der Besuche vor Ort (zwei Projekte) durchgeführte Teilnehmerlnnenbefragung zeigt eine (relativ) hohe Gesamtzufriedenheit des bisherigen Schulungsverlaufs. Einzig der Einblick in teilnehmende Unternehmen komme etwas zu kurz. Im Projekt McTronic, wo die TeilnehmerInnen sehr unterschiedliche Vorbildung haben, gibt es auch Zweifel über die Anwendbarkeit des Gelernten im Berufsalltag.

Die Heterogenität der TeilnehmerInnen stellt in den Schulungen die größte Herausforderung dar, da das Vorwissen selbst innerhalb von Personengruppen mit ähnlicher Ausbildung (z.B. HTL-AbsolventInnen) sehr unterschiedlich ist. Wenn dann noch TeilnehmerInnen aus verschiedenen Branchen aufeinanderprallen, so die Betroffenen, bedarf es einiger Anstrengungen, um alle auf ein ähnliches Wissensniveau zu bringen und eine gemeinsame Sprache zu entwickeln. Auch kommen die TeilnehmerInnen mit unterschiedlichen Erwartungshaltungen in die Schulungen. Allerdings ist es auch explizites Ziel zweier Innovationslehrveranstaltungen, ein gemeinsames Verständnis und ganzheitliches Denken der verschiedenen Bereiche (z.B. technische Bereiche, produzierende und ausführende Firmen) zu erarbeiten.

Problematisch ist auch die Terminfindung bzw. v.a. für kleine Firmen die wiederholte Abwesenheit der Teilnehmerlnnen im Betrieb („Die vielen Tage muss man sich freispielen"). Hier musste im Vorfeld viel Überzeugungsarbeit geleistet werden, damit die KMU den Mehrwert der Innovationslehrveranstaltung erkennen und zeitweise auf ihre ExpertInnen im Tagesgeschäft verzichten. Denn die KMU erkennen zwar die Notwendigkeit von Weiterbildung, können aber die Personalressourcen oft nicht bereitstellen. Nichtsdestotrotz mussten Schulungen verschoben werden, wenn Termine in nachfrageintensiven Perioden der Branche geplant waren. So kam es in der Folge zur Beantragung von kostenneutralen Verlängerungen der Projekte.

\section{Kooperationen}

In zwei Fällen liegt die Projektleitung beim wissenschaftlichen Partner, in dem Projekt, bei dem Projektleitung und wissenschaftliche Leitung von unterschiedlichen Organisationen verantwortet wird, kommt es zu einer regelmäßigen Abstimmung der Partner. Die meisten der befragten Unternehmen hatten schon im Vorfeld Kontakt zu den wissenschaftlichen Partnern, z.B. wurden kleinere Projekte durchgeführt, FH-PraktikantInnen beschäftigt bzw. auch auf langstehenden Kooperationen aufgebaut. Auch die Unternehmen kannten sich zumindest teilweise schon, einige standen auch schon in geschäftlichen Beziehungen. 
Zu Beginn der Projekte wurden Konsortialtreffen abgehalten. Diese wurden als sehr wichtig erachten, um Erwartungshaltungen zu diskutieren, gemeinsame Ziele zu formulieren sowie Vorbehalte abzubauen und Vertrauen aufzubauen („Es braucht ein paar Monate, bis man sich kennt."). Teilweise ist geplant, diese in gröBeren Abständen zu wiederholen. V.a. mit neuen Partnern wird die Wichtigkeit persönlicher Treffen unterstrichen. Sonst verläuft die Kommunikation mit den Unternehmen meist bilateral per E-Mail auf Geschäftsführerebene. In einem Projekt wurde hier ein wissenschaftlicher Mitarbeiter komplett für diese Aufgabe freigestellt, in einem anderen übernimmt ein Cluster die Terminkoordination und die Organisation der Schulungen etc. Zwei der Projekte (bei einem in Planung) setzen auf elektronische Plattformen zur Informationsverbreitung. Die Zusammenarbeit und die Kommunikation dürften in den Konsortien in allen Fällen reibungslos verlaufen.

Als größte Herausforderung wird das Zeitmanagement bzw. die Terminfindung gesehen. Dabei hat sich eine längerfristige Planung als hilfreich erwiesen sowie die Berücksichtigung von saisonalen Auftragsspitzen und die entsprechende Adaptierung des Zeitplans. So berichten die befragten Unternehmen hier v.a. von anfänglichen Schwierigkeiten, die nun ausgeräumt wurden.

\section{Wirkungen}

Alle drei Innovationslehrveranstaltungen haben noch den Großteil ihrer Laufzeit vor sich, d.h. Aussagen über Wirkungen können zum derzeitigen Zeitpunkt nicht getroffen werden. Zudem gibt Z.B. ein Interviewpartner zu bedenken, dass Innovation ein permanenter Prozess sei und es schwierig sein wird, Kausalzusammenhänge herzustellen und bestimmte Ereignisse ausschließlich der Innovationslehrveranstaltung zuzuschreiben. Es gibt aber Pläne, wie die Projektergebnisse weiterverwertet werden sollen bzw. erste Indizien, welche Auswirkungen die Innovationslehrveranstaltungen haben könnten:

- Transferprojekte: Transferprojekte bilden laut Ausschreibungsunterlage einen wesentlichen Bestandteil der Innovationslehrveranstaltungen. Diese sollen dazu dienen, das Gelernte in allen teilnehmenden Unternehmen zu implementieren. Dies können Coachings vor Ort, Vortragsreihen durch SchulungsteilnehmerInnen oder die Einführung von Technologieverbesserung sein. In den Projekten hat sich gezeigt, dass diese Transferprojekte erst nach Abschluss gewisser Kurse sinnvoll sind bzw. erst später im Projektverlauf beginnen können und wurden daher teilweise verschoben. Die Konkretisierung dieser steht daher in den meisten Fällen noch aus. In MAPO wurden daher erst 2 Transferprojekte bei einem Unternehmen gestartet (eines wird jedenfalls intern verwendet, eventuell wird daraus ein kommerzielles Produkt entstehen, ein zweites wird gerade mit "friendly 
customers" getestet). Ein Unternehmen hofft, mit dem Input der Schulungen eines seiner Produkte weiterentwickeln zu können und wird Teile der Schulungen in sein Endkundenprogramm (Schulungen für Kunden) einflieBen lassen (MAPO). Bei McTronic gab es erste interne Abstimmungen bzw. sind Transferprojekte für 2015 geplant, ein befragtes Unternehmen berichtet in diesem Zusammenhang von der Konzeption einer Schulung für interne Zwecke. Auch werden die Schulungsinhalte im Rahmen bereits bestehender interner Qualifizierungsmaßnahmen weitergetragen und thematisiert (MAPO). Im Rahmen von eINNOVATION werden erste Ideen der Unternehmen diskutiert. Die Transferprojekte sollen auch zu einer nachhaltigen Vernetzung beitragen. So ist bei MAPO geplant, eine längerfristige Kooperation mit Unternehmen zu begründen. Insgesamt ist allerdings anzumerken, dass das Konzept der Transferprojekte sowohl für die Konsortialführungen als auch die Unternehmen nicht ganz klar ersichtlich war, d.h. dass es hier Unsicherheiten über den Charakter der Projekte gegeben hat.

- Verankerung von Schulungsinhalten im Bildungsangebot: Die Innovationslehrveranstaltungen sollen zu einer nachhaltigen Etablierung von wirtschaftsnahen Themen im Qualifizierungsangebot führen. Die Planungen sind hier noch eher vage und nicht konkretisiert. So plant die FH OÖ (McTronic) aus den Schulungen praxiserprobte Programme zu designen, die in der Folge Unternehmen angeboten werden können. Dies geschieht aus einer strategischen Überlegung heraus, wonach die FH in Zukunft verstärkt Unternehmen als Nachfrager von Weiterbildungsangeboten adressieren möchte. Die TU Wien (MAPO) betont, dass die Vortragseinheiten eins zu eins in die Lehre übernommen werden können und dass sich Übungseinheiten für Spezialisierungsseminare für herausragende StudentInnen an der Universität eignen. Die Möglichkeit, Schulungen in bestimmten Bereichen anzubieten, wird zurzeit von der FH Burgenland (eNNOVATION) geprüft. Auch sollen die Erkenntnisse aus dem Projekt in die Lehre an der $\mathrm{FH}$ einfließen.

- Kompetenzerweiterung: Alle Lehrveranstaltungen sollten sich in einem Know-How-Zuwachs der teilnehmenden Unternehmen niederschlagen. In diesem Zusammenhang betonen die interviewten Konsortialführer die Wichtigkeit des branchenübergreifenden Denkens, das durch die Projekte gefördert wird. Damit sollte auch in spezialisierte Unternehmen systemisches Denken oder Denken in Wertschöpfungsketten Einzug halten bzw. eine ganzheitliche Sicht Berücksichtigung finden (,Wir können den gesamtheitlichen Ansatz perfektionieren."), um neue, bessere Produkte zu entwickeln (z.B. Softwarefirmen denken Securityaspekte mit). Dies kann auch die Kommunikation mit KooperationspartnerInnen und Kundlnnen verbessern. Zudem geben die Schulungen wertvolle Impulse und führen 
dazu, „Methoden wieder zu entdecken“ und „auf dem neuesten Stand der Technik zu sein", was in Projekten direkt angewandt und zu Effizienzsteigerungen führen kann. Auch werden wichtige Bereiche identifiziert, die bis dato "stiefmütterlich behandelt wurden". Unternehmen, die auch Vortragende stellen (MAPO), berichten von den positiven Effekten durch die Vortragstätigkeit („Sie bekommen eine höhere Klarheit bezüglich der Themen.") Die wissenschaftlichen Partner können von den Innovationslehrveranstaltungen ebenfalls profitieren, da diese die Gelegenheit bieten, neue, aktuelle Themen auf die (Forschungs-)Agenda zu setzen und hier Kompetenzen zu erweitern. Dies wird auch durch wissenschaftliche Beiträge bei Tagungen (eNNOVATION) deutlich.

- Vernetzung: Obwohl die Unternehmen meist schon Kontakte mit den wissenschaftlichen Partnern im Vorfeld der Innovationslehrveranstaltungen hatten, dürfte die Projektteilnahme die Kooperationen festigen und könnte zu weiteren gemeinsamen Forschungsprojekten führen. So steht z.B. bei zwei Unternehmen eine Vertriebskooperation in Diskussion (eNNOVATION), ein Unternehmen nimmt ein Produkt eines anderen ins Portfolio (MAPO) bzw. planen Unternehmen ein gemeinsames Entwicklungsprojekt (eNNOVATION). Zudem werden die Vorteile einer informellen Kooperation unterstrichen („Wenn es brennt, weiß ich jetzt, wo ich mich hinwenden kann").

\section{Beurteilung}

Für eine fundierte Beurteilung der Innovationslehrveranstaltungen ist es zu früh, da keines der geförderten Projekte zum Zeitpunkt der Evaluierung abgeschlossen wurde bzw. vor dem Abschluss steht. Da das Instrument hohe Mittel pro Projekt bereitstellt, sollte in einer Endevaluierung des Förderschwerpunkts ein Fokus auf die Analyse dieses Instruments gelegt werden.

Dennoch lässt sich festhalten, dass die Fördernehmer die Innovationslehrveranstaltung als Instrument begrüßen, in dem unternehmerische Weiterbildung auf höchstem Niveau („Spezialistenlevel / ) auf dem wissenschaftlich neuesten Stand erfolgen kann. Die lange Laufzeit mache das Instrument attraktiv, weil so umfassendes Know-How in der Tiefe vermittelt werden kann. Hier biete sich der Rahmen für spezialisierte Ausbildungen, die den Unternehmen das Rüstzeug geben, auf aktuelle Herausforderungen reagieren zu können. Im Rahmen der Innovationslehrveranstaltungen erfolgt der Know-How-Transfer nicht einseitig, sondern in mehrere Richtungen (von den wissenschaftlichen Partnern zu den Unternehmen, aber auch umgekehrt sowie zwischen den Unternehmen), was eine entscheidende Stärke des Instruments darstellt. Über Transferprojekte soll das erlangte Know-How ins Unternehmen getragen bzw. umgesetzt und somit nachhaltig verankert werden. In welcher Form dies geschieht, ist zu einem späteren Zeitpunkt zu untersuchen. 
Auch wird die Möglichkeit zur Netzwerkbildung - insbesondere mit den wissenschaftlichen Partnern - als Vorteil erkannt. Aber auch die daraus entstehende informelle Zusammenarbeit mit den anderen teilnehmenden Unternehmen wird als positiv hervorgehoben („Wenn man ein Problem hat, greift man auf diese Kontakte zurück.") ebenso wie die Möglichkeit zu zukünftigen Kooperationen.

Die Akzeptanz des Instruments wird auch dadurch sichtbar, dass in einem Fall eine neuerliche Einreichung bereits geplant ist und sich alle interviewten Unternehmensvertreter eine neuerliche Teilnahme an einer Innovationslehrveranstaltung vorstellen können, wenn das Thema für das Unternehmen von Relevanz ist.

Kritik gibt es nur hinsichtlich des Namens, der den umfassenden Möglichkeiten des Instruments in keiner Weise gerecht werde („Die Namensgebung ist unglücklich, das klingt nach dem kleinsten Instrument"). 


\section{Das Programmmanagement}

Das Programm Forschungskompetenzen für die Wirtschaft ist innerhalb der FFG dem Bereich Strukturprogramme zugeordnet. Es weicht von der Förderlogik der FFG im engeren Sinn ab, als in dessen Rahmen keine Forschungsprojekte sondern Qualifizierungsmaßnahmen gefördert werden. Das Programm ist relativ neu (Start: Mitte 2011) und hat noch keine hohe Bekanntheit unter den potenziellen Förderwerbern $^{7}$. Dies geht teilweise mit einem höheren Erklärungsbedarf bei den Förderwerbern im Vergleich zu den „klassischen“ FFG Programmen einher, was noch dadurch verstärkt wird, dass sich die Qualifizierungsseminare und auch die Qualifizierungsnetze explizit an Fördereinsteiger wenden.

Der vergleichsweise geringe Bekanntheitsgrad des Programms hat auch Folgen in Hinblick auf die Ausschöpfung der Fördermittel. Die für das Einsteigerformat Qualifizierungsseminare reservierten Budgets werden nicht ausgeschöpft, während die Qualifizierungsnetze fast doppelt und die Innovationslehrveranstaltungen noch deutlicher überzeichnet sind. Dies hat laut Programmmanagement mehrere Gründe. Die Unternehmen und hier insbesondere die Fördereinsteiger werden selten direkt erreicht. Dies gelingt am besten über Intermediäre (z.B. Cluster) bzw. die Universitäten bzw. Fachhochschulen. Letztere zeigen sich aber an den „größeren“ Instrumenten interessierter. Dadurch kann es zu einer „Kannibalisierung“ des geringer dotierten Instruments durch die besser dotierten kommen. Zudem berichten die wissenschaftlichen Partner davon, viel Überzeugungsarbeit bei potenziellen Fördereinsteigern leisten zu müssen.

Das Programmmanagement strebt daher an, v.a. die Qualifizierungsseminare verstärkt zu bewerben. Die bereits erfolgte Informationsoffensive bei den Universitäten und Fachhochschulen hat hier keinen messbaren Erfolg gezeigt. Daher wird in Zukunft besonderer Augenmerk auf die Intermediäre gelegt und eine gezielte Kommunikation mit Clustern, Standortagenturen etc. insbesondere in Westösterreich angeregt. Der Kontakt zur Wirtschaftskammer und zur Industriellenvereinigung wurde bereits verstärkt und hier stößt das Programm auf breites Interesse.

\footnotetext{
${ }^{7}$ Im Rahmen der Zwischenevaluierung des Programms Innovationsscheck Plus, das eine ähnliche Zielgruppe aufweist, wurden z.B. Fördernehmer durch die KMU Forschung Austria nach der Kenntnis des Programms Forschungskompetenzen für die Wirtschaft befragt. Nur $15 \%$ der Befragten kannten das Programm.
} 
Bei den Auswahlverfahren kommen folgende FFG-Modelle zum Einsatz:

- Bei den Qualifizierungsseminaren findet das Modell 1, ein verkürztes Verfahren für Kleinprojekte, Anwendung. Es handelt sich hierbei um ein Antragsverfahren, bei dem die Bewertung durch externe FachgutachterInnen vorgenommen wird und die Förderentscheidung bei der FFG liegt.

- Zur Beurteilung der Qualifizierungsnetze wird das Modell 3 herangezogen. Dies ist ein Wettbewerbsverfahren mit externem Bewertungsgremium. Die Qualität der Anträge wird durch FFG-ExpertInnen und externe Fachgutachterlnnen beurteilt, die Förderempfehlung erfolgt durch ein externes Bewertungsgremium. Die Förderentscheidung trifft das BMWFW.

- Bei den Innovationslehrveranstaltungen kommt Modell 4 zur Anwendung. Die Beurteilung der wirtschaftlichen Qualität erfolgt im Wettbewerbsverfahren durch die Jurymitglieder und die FFG-ExpertInnen, die Jury formuliert die Förderempfehlung auf der Grundlage der Anträge und eines Hearings. Die Förderentscheidung trifft wiederum das BMWFW.

Sowohl Programmmanagement als auch die befragten Jurymitglieder erachten die Auswahlverfahren und die Förderkriterien als den jeweiligen Instrumenten angemessen. Bei den Qualifizierungsseminaren hat es eine Änderung in dem Sinn gegeben, als nunmehr drei Fachgutachten statt wie in der ersten Ausschreibung nur zwei eingeholt werden, was zu klareren Entscheidungen beiträgt.

Eines der Hauptpunkte in den Diskussionen der Jury war die Frage nach der Additionalität der eingereichten Förderanträge, d.h. die Frage, ob es ein zur Förderung eingereichtes Schulungsangebot schon am Markt gibt oder nicht. Hier kam erschwerend hinzu, dass in den Anträgen teilweise keine oder eine nur unzureichende Abgrenzung zu bestehenden Angeboten vorgenommen wurde. Daher mussten oftmals Nachrecherchen durchgeführt werden.

Die Besonderheiten des Programms, d.h. dass hier nicht Forschungsprojekte sondern Qualifizierungsmaßnahmen beurteilt werden mussten, erforderte ein Umdenken bzw. die Infragestellung der eigenen Wertungen seitens der Juroren („Ich musste genau überlegen, mit welcher Brille ich auf die Dinge schaue.“, „Da muss man manches in einem anderen Licht betrachten"). Die Zusammensetzung der Jury sowie die Diskussionen in dieser wurden als sehr befruchtend wahrgenommen, ebenso wie die gemeinsame Formulierung etwaiger Auflagen.

Insgesamt wurde in Hinblick auf das Auswahlverfahren von den befragten Juroren die hervorragende Vorbereitung durch das FFG-Programmmanagement hervorgehoben. Nicht nur die Jurysitzungen, auch der ganze Prozess sei „ausgesprochen gut und strukturiert vorbereitet" gewesen. Es wurde schon im Vorfeld in Briefings, Telefonaten etc. klargestellt, wie der Prozess abläuft, und welche Punkte zu beachten sind. Auch wurde deutlich auf neue bzw. geänderte Kriterien hingewiesen. 
Der Antragsprozess verläuft meist ohne Probleme. Da und dort gibt es Schwierigkeiten mit neuen Kundlnnen, die mit dem e-call noch nicht vertraut sind, die erhöhten Unterstützungsbedarf mit sich bringen. Die Berichtsvorlagen scheinen zum Teil nicht ganz passend, da diese für Forschungsprojekte konzipiert sind. Hier könnten Adaptierungen vorgenommen werden. Bei den Anträgen der ersten Ausschreibungen wurde oftmals die qualitative Darstellung der Qualifizierungen nicht deutlich gemacht, d.h. wie viele und welche Teilnehmerlnnen welche Schulungen in welchem Ausmaß in Anspruch nehmen. Dies hat sich aber nach verstärkter Information der Antragsteller bei den zweiten Ausschreibungen deutlich gebessert. Dennoch berichten einige Unternehmen davon, dass gerade diese Vorgabe oft schwer zu erfüllen ist und dann Änderungen beantragt werden müssen. Eine Flexibilisierung würde hier den Unternehmen zugutekommen, da ja oftmals die genauen Schulungsinhalte erst festgelegt und auf dieser Grundlage die geeignetsten TeilnehmerInnen identifiziert werden können.

Die Fördernehmer und Projektpartner beurteilen die Abwicklung und Betreuung der Projekte durch die FFG fast durchwegs sehr positiv. Die Vorgaben, geforderten Unterlagen etc. seien im Großen und Ganzen adäquat zur jeweiligen Förderhöhe. Die erfahrenen Unternehmen sprechen von Erfordernissen „in üblichem Rahmen“ und v.a. die Fördereinsteiger schätzen sehr, dass die wissenschaftlichen Partner die Administration mit der FFG übernehmen können („Für uns ist das sehr unbürokratisch“, „easy going"). Auch die Konsortialführer der Innovationslehrveranstaltungen fühlen sich gut betreut und loben die kompetenten Ansprechpartnerlnnen in der FFG. Einige wenige Verbesserungsvorschläge gibt es dennoch:

- Bei den Innovationslehrveranstaltungen wurde angeregt, mehr Flexibilität hinsichtlich der Schulungsinhalte zuzulassen bzw. diese zu Beginn noch nicht detailliert festlegen zu müssen. Geplante Themen können in dynamischen Bereichen schnell „nicht mehr so interessant sein“, dagegen können andere Themen an Aktualität gewinnen.

- Das Konzept der Transferprojekte im Rahmen der Innovationslehrveranstaltungen sollte in den Ausschreibungsunterlagen klarer erklärt werden. Diese sollten auch zeitlich gegen Ende des Projekts erfolgen dürfen, wenn zumindest schon einige Schulungen stattgefunden haben.

Innerhalb der FFG hat das Programm laut Strategieabteilung inzwischen ein gutes Standing, was auch auf die gute Kommunikationstätigkeit des Programmmanagements nach innen zurückzuführen ist. Dennoch ist das Verständnis für F\&EProjekte noch immer höher und ein Programm wie Forschungskompetenzen für die Wirtschaft hat eine gewisse Sonderstellung. Dass aber das Thema Humanressourcen in der FFG insgesamt an Bedeutung gewinnt, zeigt auch die Etablierung eines Thementeams Humanressourcen. 


\section{Schlussfolgerungen}

Der Förderschwerpunkt Forschungskompetenzen für die Wirtschaft adressiert einen Problembereich der österreichischen Forschung - den Mangel an qualifizierten Fachkräften, besonders in KMU. Er ist als strukturelle Maßnahme zur Stärkung der Humanressourcen in der Wirtschaft konzipiert. Über die Höherqualifizierung des vorhandenen Forschungs- und Innovationspersonals sollen die Unternehmen in ihrer Innovationskompetenz gestärkt werden. Die Themen der Qualifizierung sollen wirtschaftsnah und zukunftsrelevant sein und in Abstimmung mit den Unternehmen festgelegt werden. Forschungskompetenzen für die Wirtschaft schließt damit eine Lücke im Förderportfolio der FFG. Die Zielsetzung des Programms entspricht der FTI-Strategie der Bundesregierung.

Die drei Instrumente Qualifizierungsseminare, Qualifizierungsnetze und Innovationslehrveranstaltungen mit tertiärem Charakter sind den operativen Zielen des Kompetenzaufbaus, der Kompetenzvertiefung und der Kompetenzerweiterung zugeordnet und können in Hinblick auf Länge der Projekte und Anspruchsniveau als hierarchisch angeordnet betrachtet werden. Dies zeigt sich auch in den jeweils adressierten Zielgruppen. Die Qualifizierungsseminare sind als kurzfristiges Einsteigerformat konzipiert und richten sich an FTEI-Einsteiger. Im Rahmen des mittelfristigen Formats der Qualifizierungsnetze für FTEI-Einsteiger und technologisch kompetente Unternehmen soll Wissen vertiefend aufgebaut werden. Technologisch kompetenten Unternehmen und forschungsorientierten Unternehmen stehen auch die längerfristigen Innovationslehrveranstaltungen zur Verfügung, die auf Hochtechnologiefelder fokussieren sollen. Das unterschiedliche Anspruchsniveau der Instrumente zeigt sich auch in den Erfolgsquoten der Einreichungen. Diese liegen bei den Qualifizierungsseminaren bei über $70 \%$, bei den Qualifizierungs netzen bei über $50 \%$ und bei den Innovationslehrveranstaltungen bei $38 \%$.

Allen drei Instrumente immanent ist die Charakteristik einer Qualifizierung in Netzwerken. Damit wird neben der Qualifizierung durch externe ExpertInnen über den Erfahrungsaustausch auch „peer learning“ unterstützt und ein Beitrag zur Netzwerkbildung von Unternehmen geleistet, was von den Unternehmen auch als bedeutender Mehrwert wahrgenommen wird. Insgesamt kann das Konzept des Förderschwerpunkts Forschungskompetenzen für die Wirtschaft somit als gelungen betrachtet werden. 
Die einzelnen Instrumente können wie folgt beurteilt werden:

- Die Qualifizierungsseminare haben sich sowohl in ihrer Konzeption als auch in ihrer praktischen Ausgestaltung als Einsteigerformat bewährt. $50 \%$ der in diesem Rahmen geförderten Unternehmen sind Neukunden der FFG. Auch ist der Anteil an Kleinunternehmen in diesem Format am höchsten. Die Seminare entsprechen den Bedürfnissen der Unternehmen und behandeln zukunftsrelevante Themen, da schon oft im Vorfeld Weiterbildungsbedarf bei den Unternehmen identifiziert wurde, aber auch ein mangelndes Angebot an geeigneten Qualifizierungsmaßnahmen.

Durch die Teilnahme am Qualifizierungsseminar kam es bei den Unternehmen zu einer Stärkung ihrer Innovationskompetenz, was sich in Wettbewerbsvorteilen ausdrückt. Es wurde Verständnis für Innovationsprozesse entwickelt sowie die Kommunikation mit Kundlnnen und Kooperationspartnern verbessert. Viele Unternehmen konnten aufbauend auf dem erworbenen Wissen und durch die Anwendung neuer Technologien ihre Produkte/Dienstleistungen verbessern bzw. ihr Produktportfolio erweitern. Auch sind weitere (geförderte) F\&E Aktivitäten initiiert worden bzw. haben die Unternehmen nun verstärkten Zugang zu wissenschaftlichen Einrichtungen. Die wissenschaftlichen Partner nützen die Erfahrungen aus den Seminaren für die Weiterentwicklung der Lehre und planen studentische Projekte in Unternehmen. Somit können durch die Qualifizierungsseminare auch Barrieren abgebaut und neue Kommunikationskanäle zwischen Wissenschaft und Wirtschaft, und insbesondere KMU, geöffnet werden.

Das Instrument der Qualifizierungsseminare ist als sehr geeignet zur Kompetenzsteigerung von KMU anzusehen. Erfolgsfaktoren sind die maßgeschneiderte Anpassung der Schulungsinhalte, der Anschluss an den aktuellsten Stand der Forschung und der Erfahrungsaustausch mit anderen Teilnehmerlnnen.

- Die Qualifizierungsnetze erweisen sich als fortgeschrittenes Instrument der Weiterbildung, das ein mittelfristiges Committment der beteiligten Partner verlangt. Unter den beteiligten Unternehmen befinden sich mit $36 \%$ daher auch weniger FFG-Neulinge als bei den Qualifizierungsseminaren. Neben der Verbreiterung des Know-Hows der Projektpartner steht hier der Vernetzungsgedanke im Vordergrund. Dabei streben einige Netzwerke auch eine branchenübergreifende Zusammenarbeit an. Dies hat zur Folge, dass es zu einer größeren Heterogenität der Teilnehmerlnnen kommt. AuBerdem zeigen sich Engpässe in Hinblick auf die Vereinbarkeit mit dem betrieblichen Alltag, da insbesondere kleine Unternehmen oft nur schwer ihre Fachkräfte für Weiterbildungsmaßnahmen über einen längeren Zeitraum abstellen können. So wird das Instrument von vielen Beteiligten auch als „fordernd" bezeichnet. 
Die teilnehmenden Unternehmen berichten von einer Erhöhung ihrer Innovationskompetenz, die sie teilweise durch interne Schulungen noch verstärken wollen. Dies schlägt sich u.a. in einem souveräneren Auftreten bei den Kundlnnen nieder, führt aber auch zu Motivationsschüben bei den MitarbeiterInnen. Produkte bzw. interne Unternehmensprozesse wurden optimiert bzw. neue Produkte entwickelt. Netzwerkaktivitäten sind auch nach dem Auslaufen mancher Projekte zu beobachten, es wurden z.B. Vereine gegründet oder die Partner engagieren sich in bestehenden Netzwerken. Besonders hervorzuheben ist die nachhaltige Verankerung von Qualifizierungsangeboten (z.B. akademischer Lehrgang und Masterlehrgang, Tagesschulungen, Inhouse-Seminare), die auf Basis der Erfahrungen im Qualifizierungsnetz konzipiert wurden.

Die Qualifizierungsnetze genießen hohe Akzeptanz bei den Fördernehmern, was sich nicht zuletzt in der (geplanten) Teilnahme an weiteren QNProjekten ausdrückt. Als Stärken des Instruments werden die Anpassung der Schulungsinhalte an die Bedürfnisse der Unternehmen, die Kompetenz der Vortragenden und der Vernetzungsaspekt gesehen. Auch die Möglichkeit der Diskussion mit Unternehmen anderer Branchen, die Bearbeitung von Schnittstellen und die Verzahnung von Wissenschaft und Praxis werden positiv hervorgehoben. Dies führt aber zur Herausforderung, Unternehmen verschiedener Bereiche zusammenzuführen und zu koordinieren.

- Das anspruchsvollste Instrument Innovationslehrveranstaltungen spricht naturgemäß nur wenige Fördereinsteiger an (17\% der Unternehmen sind Neukunden der FFG). Bis dato wurden insgesamt nur drei Innovationslehrveranstaltungen gefördert, wobei keine zum Zeitpunkt der Evaluierung abgeschlossen ist. Die Kompetenzerweiterung erfolgt in Schlüsseltechnologien bzw. in Themenfeldern, die für die Unternehmen stark an Bedeutung gewinnen. In einem Fall steht das branchenübergreifende Systemdenken zur Bewältigung zukünftiger Herausforderungen im Vordergrund. In allen drei Fällen dürfe das jeweilige Projekt eine große Lücke im Angebot schließen, da diese Spezialthemen bzw. sehr dynamischen Bereiche nicht in Standardschulungen behandelt werden. Daher sprechen die Unternehmen auch von einem sehr attraktiven Schulungsangebot.

Die Konkretisierung der Transferprojekte, die dazu dienen sollen, das Gelernte in den Unternehmen zu implementieren, steht in den meisten Fällen noch aus ebenso wie die nachhaltige Verankerung von Schulungsangeboten. Hier gibt es erste Absichtserklärungen (z.B. Konzeption von Weiterbildungsprogrammen / Schulungen für Unternehmen, Vortrags- und Übungseinheiten können in die Lehre an der Universität übernommen werden). Eine Kompetenzerweiterung wird erwartet, v.a. hinsichtlich der Entwicklung einer ganzheitlichen Sicht über Branchen hinweg. Zudem dürften die Innovationslehrveranstaltungen bestehende Kooperationen festigen. 
Die bis dato durchgeführten Schulungen stoßen auf positive Resonanz der TeilnehmerInnen, die Fördernehmer begrüßen das Instrument, da hier unternehmerische Weiterbildung auf höchstem Niveau und spezialisierte Ausbildungen stattfinden können. Eine entscheidende Stärke des Instruments ist der multilaterale Know-How-Transfer (von den wissenschaftlichen Partnern zu den Unternehmen, aber auch umgekehrt sowie zwischen den Unternehmen). Damit einher geht auch die verstärkte Netzwerkbildung und informelle Zusammenarbeit.

Allen Instrumenten gelingt es entsprechend ihrer Ausrichtung nicht nur Förderneulinge anzusprechen, sondern diese auch nachhaltig an F\&E heranzuführen, was sich an den Beteiligungen der FFG-Neulinge an weiteren durch die FFG geförderten Projekten zeigt sowie an den Folgeaktivitäten aller im Rahmen von Forschungskompetenzen für die Wirtschaft geförderten Unternehmen. Rd. jeder Vierte Fördernehmer nahm seit September 2013 andere FFG-Förderungen in Anspruch (v.a. im Rahmen der Basisprogramme und von Comet und Talente).

Aufgrund dieser Befunde wird eine Fortführung des Programms zum jetzigen Zeitpunkt empfohlen. Dies betrifft insbesondere die Instrumente Qualifizierungsseminare und Qualifizierungsnetze, die Innovationslehrveranstaltungen können noch nicht umfassend beurteilt werden. Da es sich hier um das langfristigste Instrument handelt, gilt es aber, diesem ausreichend Zeit zu gegeben, um seine Wirkungen entfalten zu können. Erste Optimierungsvorschläge konnten wie folgt identifiziert werden:

- Das Konzept des Förderschwerpunkts kann als gelungen angesehen werden. Teilweise sollten aber die Zielformulierungen überdacht werden, v. a hinsichtlich der Abgrenzung von strategischen und operativen Zielen und dessen, was das Programm leisten kann (betrifft: Erhöhung der sektoralen Mobilität). Zudem sind die im Programmdokument aufgelisteten Indikatoren teilweise vage formuliert, kaum operationalisierbar bzw. relational und die Aussagekraft muss teilweise angezweifelt werden. Eine Überarbeitung der Indikatoren (Vorschläge siehe Kap. 2), nicht zuletzt in Hinblick auf eine weitere Evaluierung, wird daher empfohlen.

- Die Abgrenzung zwischen Qualifizierungsnetzen und Innovationsveranstaltungen scheint nicht ganz klar. Der Titel "Innovationslehrveranstaltungen“ trägt zudem dem langfristigen Charakter der Maßnahme über mehrere Semester hinweg nicht Rechnung. Hier könnte nach Alternativen gesucht werden, der auch die Abgrenzung der zwei Instrumente verdeutlicht, z.B. "Qualifizierungsprogramm". 
- Inwieweit sich die Instrumente Qualifizierungsnetze und Innovationsveranstaltungen unterscheiden, steht auch mit den Wirkungen letzterer in Zusammenhang. Diese können zum gegebenen Zeitpunkt noch nicht abgeschätzt werden, da keine Innovationslehrveranstaltung bis dato abgeschlossen wurde. Die Berechtigung dieses hoch dotierten Instruments wird v. a. auch davon abhängen, inwieweit es in der Folge zu einer Etablierung von neuen Themen im Qualifizierungsangebot und einer Verankerung unternehmensrelevanter Lehr- und Forschungsschwerpunkte an Universitäten und FHs kommt, v. a. auch im Vergleich zu den Qualifizierungsnetzen. Auch den Transferprojekten wird bei der Beurteilung des Förderinstruments Bedeutung zukommen. Ebenso sind einige Kooperationsprojekte in Planung. Inwiefern diese Vorhaben umgesetzt werden und es zu einer nachhaltigen Etablierung der behandelten Themen im Qualifizierungsangebot kommt, ist in einer späteren Evaluierung zu prüfen, bei der die Innovationslehrveranstaltungen in den Fokus gerückt werden sollten.

- Bei den Innovationslehrveranstaltungen war den Fördernehmern nicht ganz klar, welchen Charakter Transferprojekte haben sollten. Hier sollten die Ausschreibungsunterlagen überarbeitet werden und deutlich machen, welche Art von Projekten erwartet wird.

- Bei den Qualifizierungsseminaren und auch bei den Innovationslehrveranstaltungen erwähnten einige Unternehmen in den Interviews, dass sie am Seminar auch ohne Förderung der Personalkosten teilgenommen hätten. In einer späteren Evaluierung sollte die Additionalitätswirkung der monetären Förderung der Personalkosten für die Unternehmen systematisch für alle drei Instrumente im Rahmen einer Unternehmensbefragung überprüft und hier gegebenenfalls Anpassungen vorgenommen werden.

- Der Frauenanteil der Teilnehmerlnnen in allen drei Instrumenten spiegelt die Unterrepräsentanz von Frauen in Naturwissenschaft/Technik und Forschung im Allgemeinen und in bestimmten Branchen und Bereichen im Besonderen wider. Da hier vorhandenes Personal geschult wird, limitiert zudem die Personalstruktur der Unternehmen den Handlungsspielraum. Maßnahmen zur Steigerung der Sensibilität in Genderfragen bei den beteiligten Unternehmen könnten im Rahmen eines solchen Programms noch forciert werden. 
- Die Fördermittel, die für die Qualifizierungsseminare bereit stehen, werden nicht ausgeschöpft. Da sich das Format an Fördereinsteiger richtet und auch für diese Zielgruppe sehr geeignet scheint, gilt es, zielgruppengerechte Mobilisierungsstrategien, zu erarbeiten. Insbesondere Intermediäre, aber auch Interessensvertreter, können hier eine unterstützende Rolle einnehmen und zur Bekanntheit des Instruments beitragen sowie Überzeugungsarbeit in Hinblick auf die Sinnhaftigkeit von Weiterbildungsmaßnahmen im Unternehmen und Netzwerkbildung leisten.

- Der Förderschwerpunkt als Ganzes ist noch nicht sehr bekannt in der Community und bei potenziellen Fördernehmern. Daher sollte insgesamt Augenmerk auf die Kommunikationsaktivitäten gesetzt werden, damit sich Forschungskompetenzen für die Wirtschaft etablieren kann. PR-Maßnahmen könnten mit den anderen Humanressourcenprogrammen (Förderschwerpunkt Talente) der FFG koordiniert werden. Aber auch im Zusammenhang mit anderen Einsteigerformaten bzw. Formaten, die sich besonders an KMU richten (im KMU-Paket der FFG), sollte das Programm in Erscheinung treten.

- Die Steigerung des Bekanntheitsgrads könnte auch durch eine bessere Vernetzung mit und Verankerung in der Qualifizierungsszene erreicht werden. Kooperationen mit Institutionen, die die Qualifizierung von ArbeitnehmerInnen fördern (z.B. AMS), könnten weitere Kommunikationskanäle eröffnen und Synergieeffekte generieren. 
KMU Forschung Austria

Austrian Institute for SME Research 